$A N L=6075$

Physics and Mathematics

(TID-4500, 15th Ed.)

AEC Research and

Development Report

\author{
ARGONNE NATIONAL LABORATORY \\ P. O. Box 299 \\ Lemont, Illinois
}

\title{
FUNDAMENTAL COMPONENTS \\ OF THE \\ "PACE" ANALOG COMPUTER
}

by

William E. Scott

November, 1959
Operated by The University of Chicago under
Contract $W-31-109-e n g-38$




\section{DISCLAIMER}

This report was prepared as an account of work sponsored by an agency of the United States Government. Neither the United States Government nor any agency Thereof, nor any of their employees, makes any warranty, express or implied, or assumes any legal liability or responsibility for the accuracy, completeness, or usefulness of any information, apparatus, product, or process disclosed, or represents that its use would not infringe privately owned rights. Reference herein to any specific commercial product, process, or service by trade name, trademark, manufacturer, or otherwise does not necessarily constitute or imply its endorsement, recommendation, or favoring by the United States Government or any agency thereof. The views and opinions of authors expressed herein do not necessarily state or reflect those of the United States Government or any agency thereof. 


\section{DISCLAIMER}

Portions of this document may be illegible in electronic image products. Images are produced from the best available original document. 


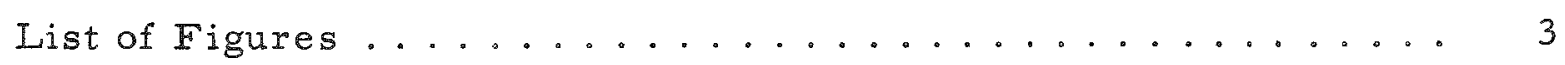

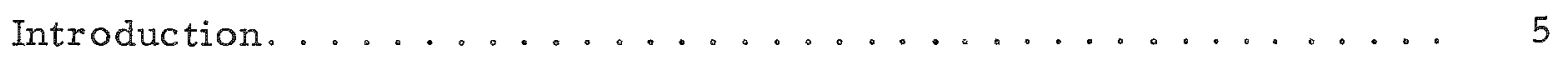

Equipment Complement..................... 7

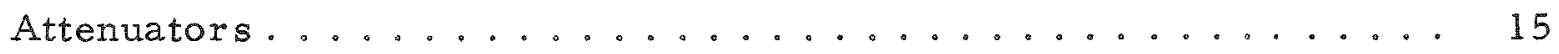

Description of Control Panel ................ 17

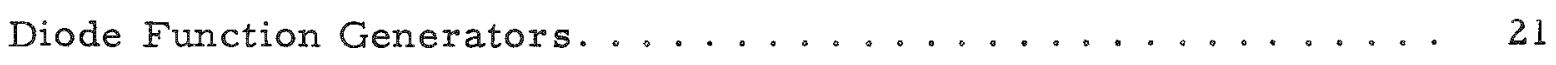

Electronic Multipliers. . . . . . . . . . . . . . . 24

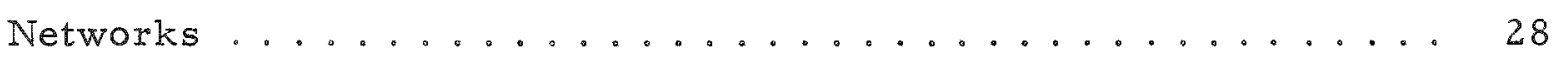

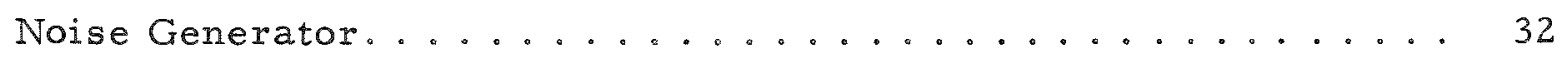

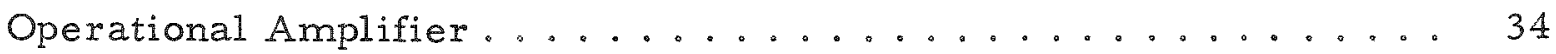

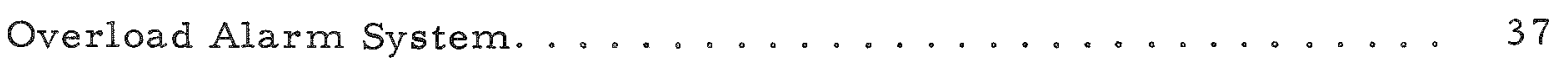

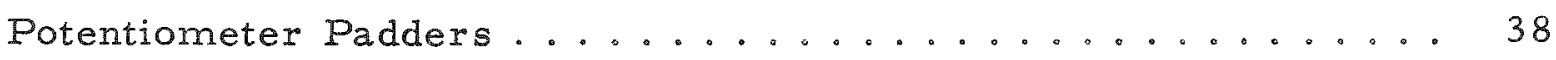

Power Supplies ........................... 42

Servo Multipliers......................... 44

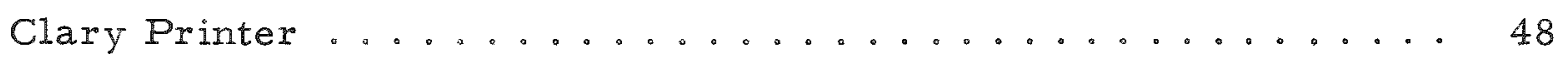

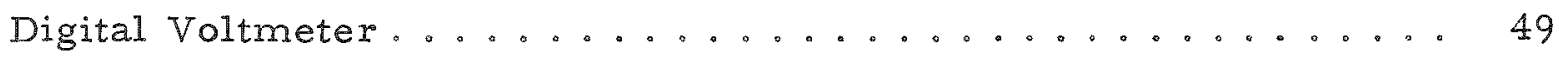

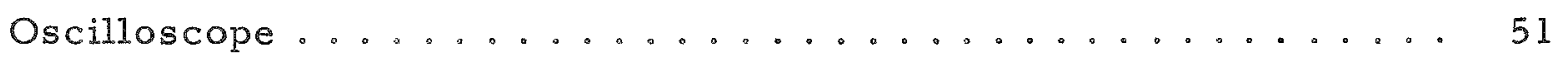

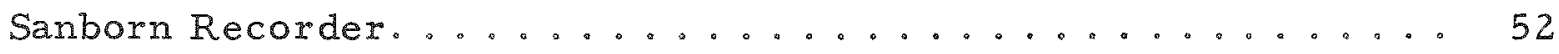

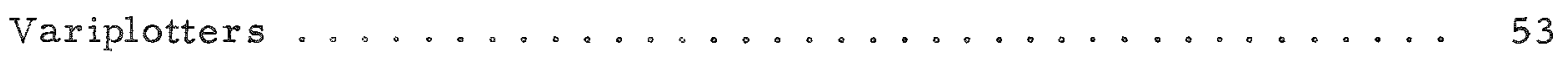

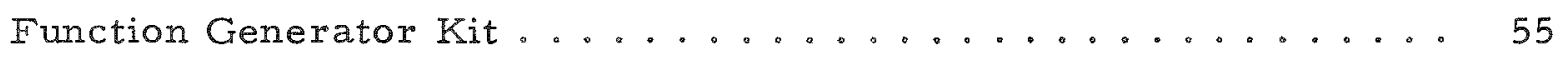




\section{LIST OF FIGURES}

No

Title

Page

1. Symbols ........................... 11

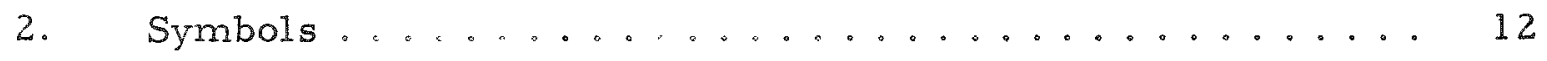

3. Component Layout Diagram ............... 13

4. Pre-Patch Panel Diagram ................ 14

5. "Initial Condition" Attenuator.............. 15

6. Grounded Coefficient Attenuator .............. 16

7. Ungrounded Coefficient Attenuator............. I6

8. Control Panel Diagram .................. 20

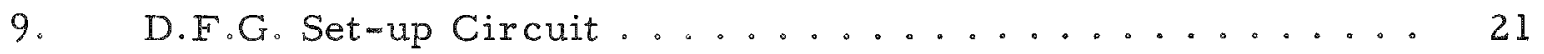

10. Sample D.F.G. Curve ................. 22

11. Block Diagram of Electronic Multiplier .......... 27

12. Electronic Multiplier Symbols ............. 27

13. Combination Amplifier Network ............. 29

14. Summing Amplifier Network. .............. 31

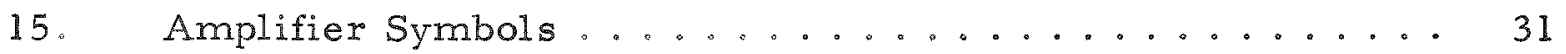

16. Block Diagram of Noise Generator ............ 32

17. Amplifier Block Diagram............... 34

18 Summing Amplifier. ..................... 35

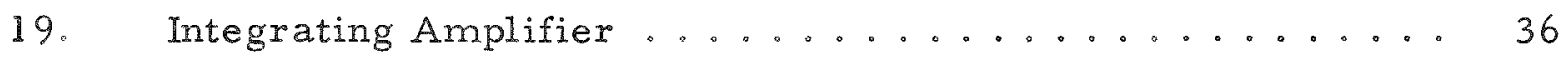

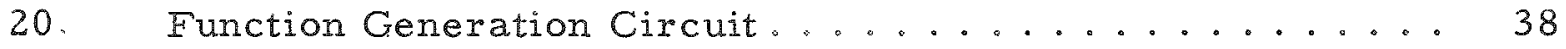

21. Sample Curve for Pot Padder Set-up ............ 39

22. D.F.G. and Pot Padder Control Panel............. 41

23. Reference Power Supplies .................. 42

24. Servo Multiplier Pots .................. 45

25. Servo Pot Terminals................. 46

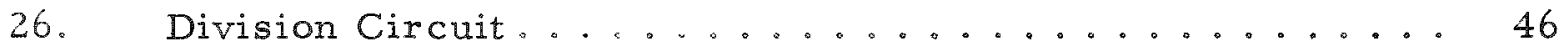

27. Block Diagram of Digital Voltmeter............ 50 


\section{FUNDAMENTAL COMPONENTS \\ OF THE \\ "PACE" ANALOG COMPUTER}

by

William E Scott

\section{INTRODUCTION}

An electronic Analog Computer is a device that uses voltages to represent physical variables, such as weight, temperature or area. The electronic circuits of the computer system must be capable of addition, subtraction, multiplication, integration, generating functions of machine variables and generating the time integral of a machine variable. The in formation that must be presented to the machine is a group of equations that describe the problem and the set of numerical values of constants and parameters of the problem

The basic computing components of the machine are the attenuators and the operational amplifiers. The attenuators provide the scaling factors and the operational amplifiers perform the arithmetic functions of addition. subtraction and integration The special computing components are diodes for simulating limitıng, backlash or hysteresis. The tapped multiplier cups and diode function generators are used for representing nonlinear functions.

The cholce between an analog computer and a digital computer is dependent upon the problem that is to be solved. The analog serves the engineer as a model of the physical system that is being designed. The digital computer can be programmed to obtain as high a degree of accuracy as desired, while the analog is accurate only to four decimal places.

The results of the analog computer are normally represented graphically as a continuous plot of the variable quantities

The most important application of the analog is in the study of control systems and missile simulation. They are also being used in nuclear reactor simulation, heat transfer problems and in the analysis of power distribution systems.

The analog computer used in this installation was manufactured by Electronic Associates, Inc, The installation consists of two consoles and a test rack. Each console is made up of 56 operational amplifiers, 5 electronic multipliers, 5 diode function generators, 1 noise generator, 8 pot padders, 80 attenuators and the recording equipment. It is possible to "slave" one console to the other for problems that require more equipment than is available on the one console 
The test rack is provided for the purpose of checking the components without causing any loss of operating time on the consoles.

The manufacturer of the computer has published maintenance and operating manuals for the individual components and the computer. These manuals are the references for the material in this report. 
EQUIPMENT COMPLEMENT

Attenuators:

There are 80 attenuators available on each console. The first twenty are the "Initial Condition" attenuators and are used to produce a constant source of voltage when connected to the \pm 100 -volt reference supply. The other 60 attenuators are the "Coefficient" attenuators and are used to multiply a varying voltage by a constant less than one. Fifty-two of the coefficient attenuators are grounded normally in the machine and eight coefficient attenuators are left ungrounded; they have all of their terminals brought out to the pre-patch panel.

Boost Resistors:

There are twelve boost resistors in the computer. The boost resistors have their upper terminal brought out to the pre-patch panel and their lower terminal connected to the +300 -volt supply. When the boost resistor is connected to the output of the amplifier, the plate resistance of the output stage of the amplifier is reduced. This allows the amplifier to supply more power to the output. Using the boost resistors results in a slight reduction of gain.

Check Amplifier:

The check amplifier acts as a summer with each individual amplifier, when connected as an integrator, so that it is possible to sum the total voltages at the summing junction.

\section{Clary Printer:}

The printer is used to print the outputs of all of the components, such as the amplifiers, servo and electronic multipliers, attenuator outputs and settings, etc. The printer will print automatically by depressing the "Auto" pushbutton on the control panel or it will print one output at a time by depressing the "Print" pushbutton on the control panel. The printer receives its signal from the digital voltmeter; (D.V.M.); therefore the D.V.M. must be operating before the printer will print.

\section{Control Panel:}

The control panel contains the off-on switch for the entire computer, the operate control switch, the signal selector pushbutton and the vacuum tube voltmeter that is used for monitoring the outputs of the components. 
Digital Voltmeter:

The digital voltmeter is used to set the attenuators, and to measure and display the operating voltages within the computer.

Diodes:

There are four diodes, with their terminals brought out to the prepatch panel, in the computer. These diodes are used in conjunction with the operational amplifiers for the representation of non-linear effects, such as back-lash, limiters, saturation, etc.

Diode Function Generators:

There are five diode function generators available on each computer. The generators are used to produce arbitrary functions of a variable. They are located in the servo rack.

Electronic Multipliers:

The electronic multipliers are used to multiply or divide two varying quantities by another. There are five electronic multipliers connected to each console. The electronic multipliers are capable of multiplying at much higher frequencies than the servo multipliers.

Function Switches:

The three function switches available are located on the control panel of each console. The double-pole double-throw switches have all of their contacts terminated on the pre-patch panel. The switches are used to provide switching for any desired purpose.

Noise Generator:

There is one electronic noise generator installed in each console and it is used to provide a source of band-limited random noise.

Operational Amplifiers:

The operational amplifiers are used to (1) form the sum of up to seven voltages, (2) invert the sign, (3) multiply the input variables by 1,5 , or 10 , and (4) to produce the integral of up to seven voltages with time. There are 56 amplifiers available on each console. The first 28 amplifiers have seven inputs and can be used as either summers, inverters, or integrators, depending upon the location of the bottle plug at the top of the amplifier position on the pre-patch panel. Amplifiers 29 through 56 have provisions for five inputs and can be 
used as summers or inverters only. The amplifier can be used as a high-gain amplifier by removing the bottle plug and wiring the input directly into the grid of the amplifier.

Oscilloscope:

The "2l-inch" oscilloscope provides a visual means of checking the computer results and the outputs of the components. It is extremely useful in troubleshooting the computer and determining the amount of noise in the components.

Overload Alarm:

The overload alarm system provides an audible and visual indication of overloading in any amplifier or servo in the computer. When the "Auto-Hold" switch on the control panel is thrown, the auto-hold circuits are energized and the computer will be automatically switched to the hold condition whenever any overload occurs.

Potentiometer Padders:

The "Pot Padders" are used in conjunction with the servo multipliers for the generation of arbitrary functions of a variable. There are eight "Pot Padding" units available on each console.

Rate Test:

The rate test feature provides a method of checking the rate of integration of each of the integrators. This is accomplished by applying $\pm n$ volts to all of the integrators simultaneously for a period of time, which is determined by the rate test potentiometer, and then checking the outputs of the integrators on the digital voltmeter. ( 100 volts $\leq n$ $\leq+100$ volts)

Recorder (Sanborn):

The Sanborn recorder provides four channels for recording the variations of computer voltages against time. It is very useful for problems where it is desirable to compare from one to four results over the same period of time.

Reference Supplies:

The $+100-$ volt and the - 100-volt power supplies provide a wellregulated reference voltage for the computer signal voltages. The reference supplies are terminated on the pre-patch panel for use with the servo multipliers, attenuators and various other uses. The reference voltages can be removed from the pre-patch panel by depressing the bar on the patch panel bay. 
Relays:

There are ten relays in the computer; all have their contacts brought out to the pre-patch panel. These relays are used for switching operations which are controlled by problem variables.

Repetitive Operation:

The repetitive operation provides for repeated integration for any part or all of a problem result.

Servo Multipliers:

There are provisions for ten servo multipliers on each console. The servos are used to provide the product of up to five variables with any sixth. When the servos are used with operational amplifiers it is possible to produce the quotient of up to five variables with any sixth.

Variplotters:

The seven variplotters in this installation are used for the graphical representation of one signal voltage against another. 


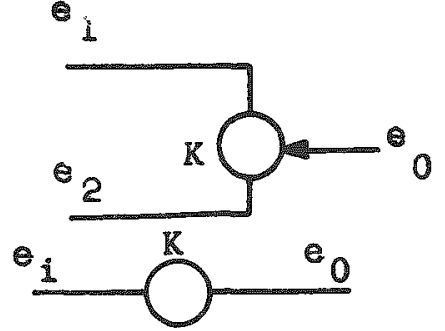

ATTENUATORS

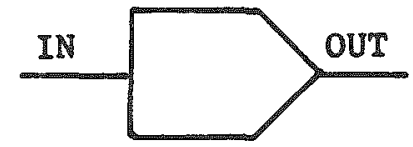

DIODE FUNCTION GENERATORS

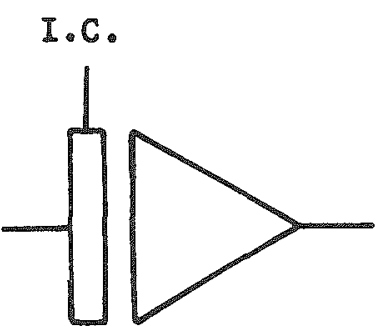

INTEGRATOR

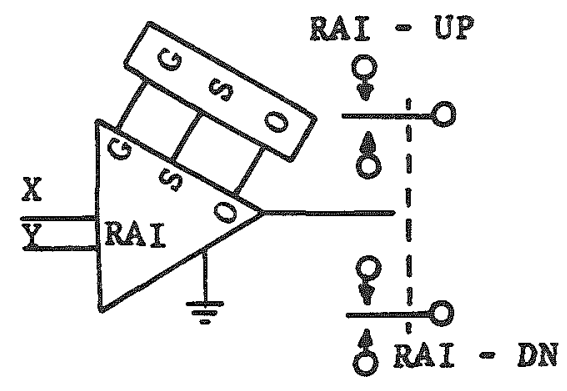

RELAY AMPLIFIER
$1 \mathrm{mfd}$

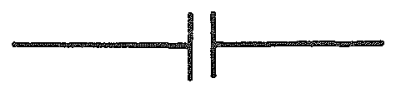

CAPACITANCE

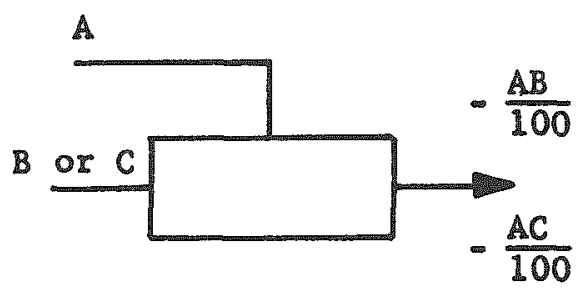

ELECTRONIC MULTIPLIER

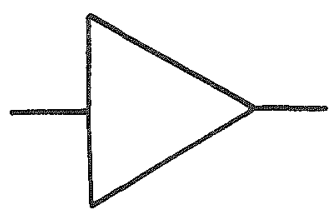

SUMMING

AMPLIF IER<smiles>c1ccccc1</smiles>

RECORDER
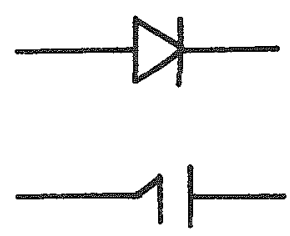

DIODES

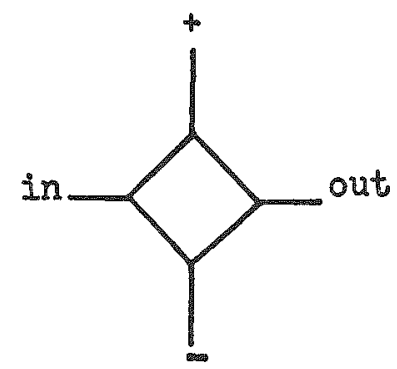

LIMITER

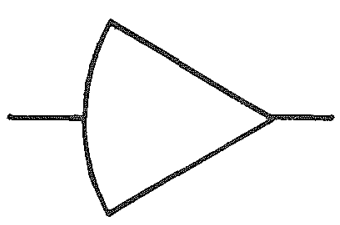

HIGH GAIN AMPLIF IER

1 M OHMS

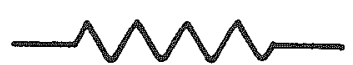

RESISTOR

Figure 1

SYMBOLS 


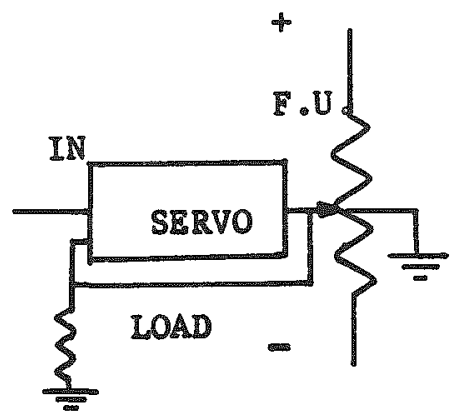

SERVO

(1) 1

TRUNKS

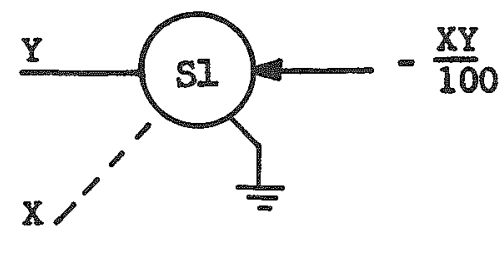

SERVO POT

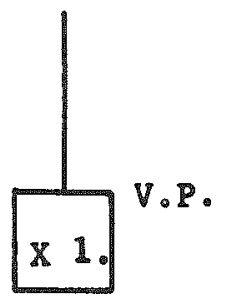

VARIPLOTTERS

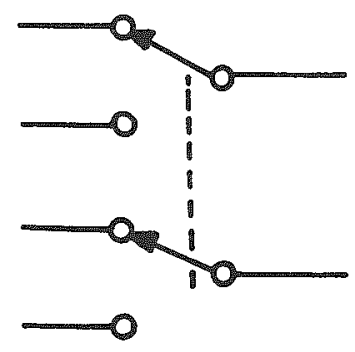

FUNCTION

SWITCHES

Figure 2

SYMBOLS 


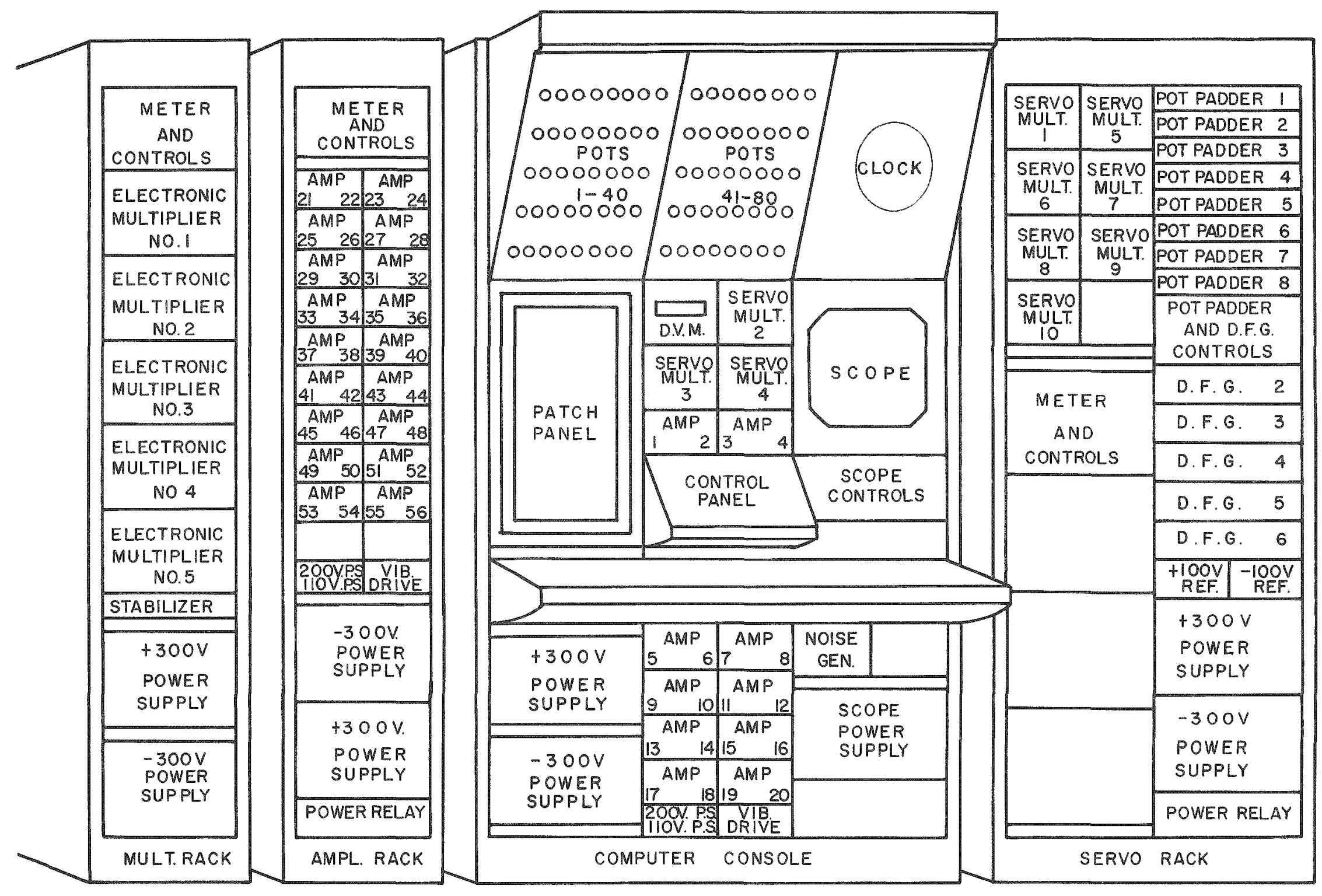

Figure 3 


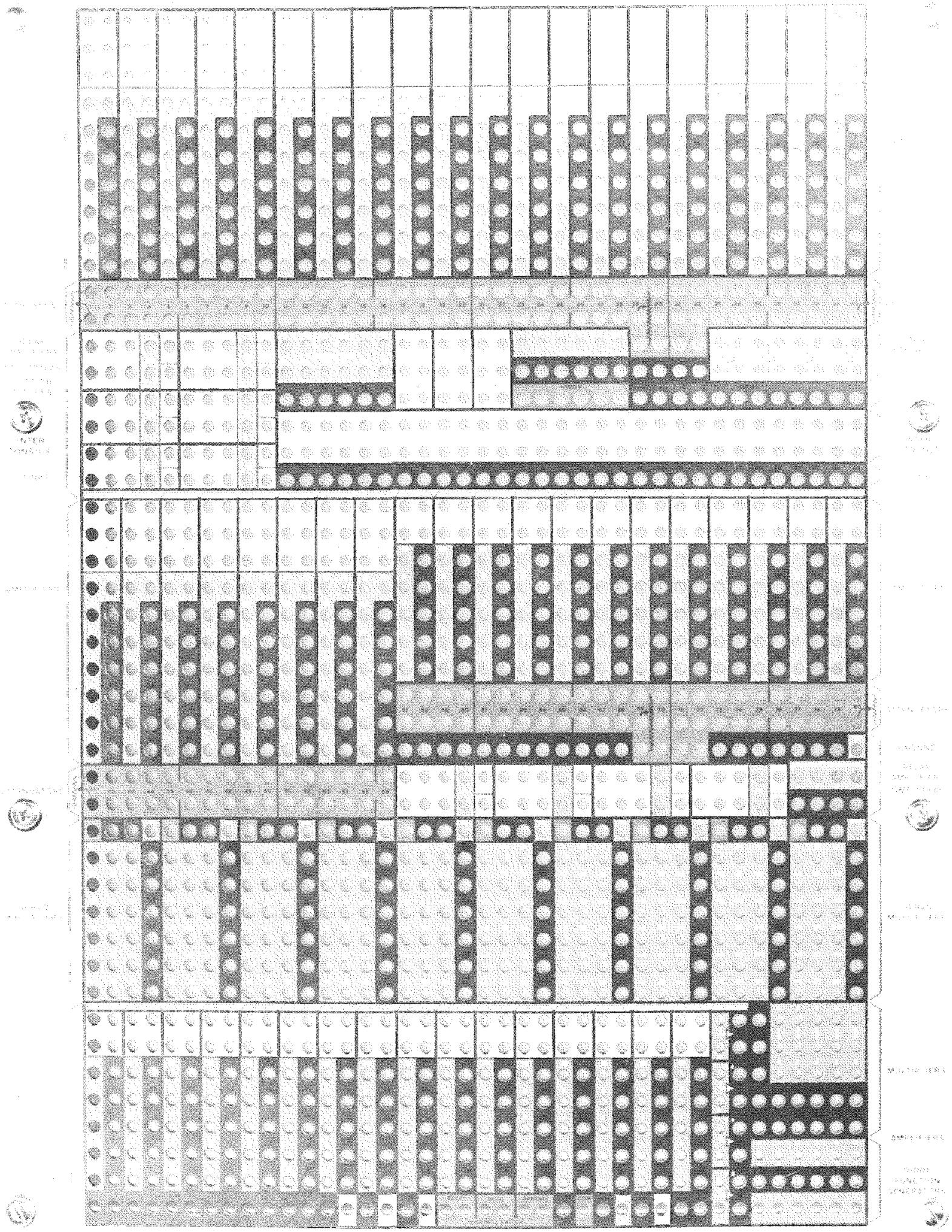

Figure 4

DIAGRAM OF PRE-PATCH PANEL 


\section{ATTENUATORS}

The attenuators serve the purpose of multiplying the variable voltage by a constant less than one and of providing a constant source of voltage, when connected to the $+100-v$ or $-100-v$ reference supply, for the "Initial Condition" of the integrators.

The attenuators are ten-turn, 30,000-ohm, helical wire-wound pots with an excellent linearity of 0.05 per cent full scale. The arm of the attenuators is fused for protection of the attenuator.

The first 20 attenuators are used for providing a constant source of voltage for the "Initial Condition" of the integrators. Each of these attenuators is associated with a three-position switch which connects the top of the attenuator to either the $+100-v$ reference supply, $-100-v$ reference supply or to the patch panel. The bottom of these attenuators is grounded in the machine. The outputs of the first 20 attenuators is monitored by means of the pushbutton signal selector switches on the control panel. Note: Attenuator number 20 is selected by depressing "pots 0,0 ." The circuit for the "Initial Condition" attenuators is shown in Figure 5.

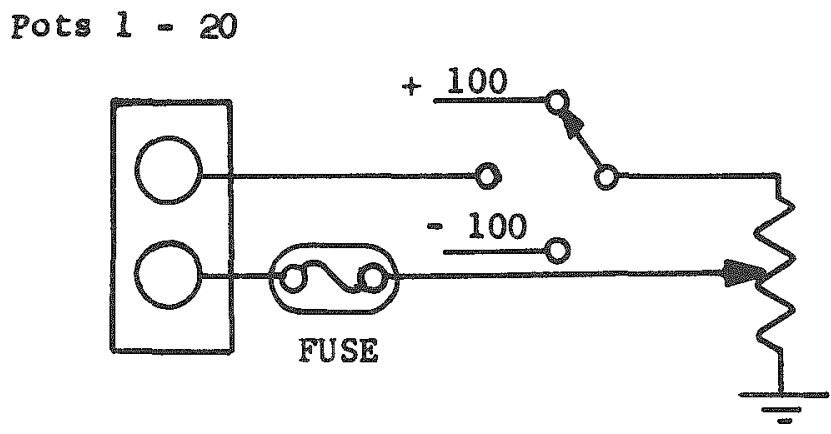

Figure 5

\section{"INITIAL CONDITION" ATTENUATOR}

The attenuators $21-80$ are the coefficient attenuators. The threeposition switch associated with each of the coefficient attenuators is a center position, spring-loaded switch. When this switch is held in the "Coef." position, the +100-v reference supply is connected to the top of the attenuator and the arm is connected to the digital voltmeter. Holding the switch in the "out" position the arm is connected to the digital voltmeter and output of the attenuator will be read.

Attenuators 29 through 32 and 69 through 73 are left ungrounded and the lower terminals of these attenuators are brought out to the patch 
panel. All of the other coefficient attenuators have their lower terminals grounded in the machine. The diagrams for the grounded and ungrounded coefficient attenuators appear in Figures 6 and 7.

$+100$

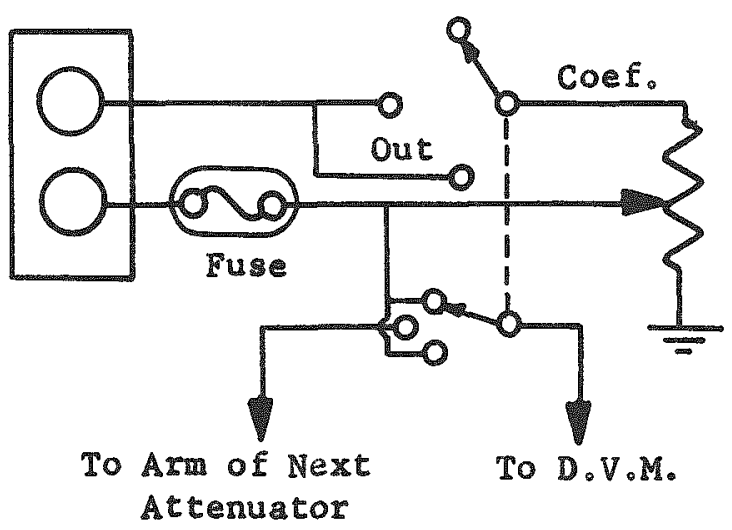

Figure 6

GROUNDED COEFFICIENT ATTENUATOR
$+100$

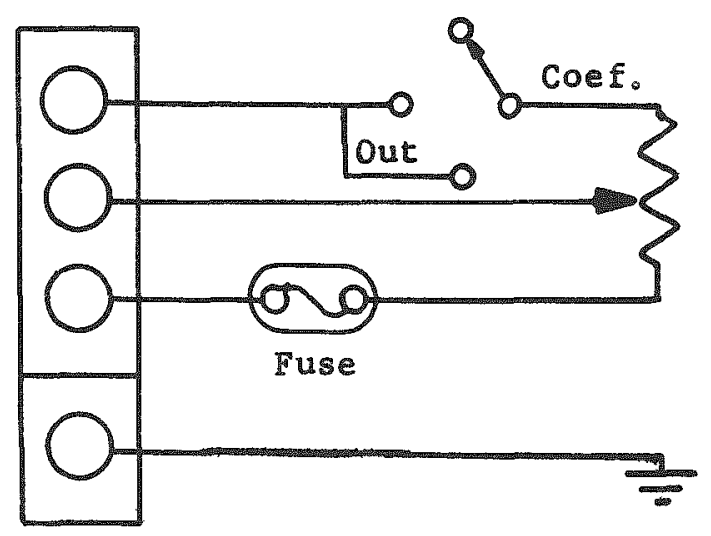

Figure 7

UNGROUNDED COEF FICIENT ATTENUATOR

The effect of amplifier loading on the attenuators causes a "loading error." Therefore the coefficient attenuators should be set under load or connected into the circuit. 


\section{DESCRIPTION OF CONTROL PANEL}

(1) SIGNAL SELECTOR PUSHBUTTONS: Selects the signal to be read on D.V.M. or the V.T.V.M., such as the outputs of the amplifiers, multipliers, Diode Function Generators, etc.

(2) PRINT BUTTON: Depressing this button causes the Clary printer to make one print.

(3) DIGITAL VOLT METER SWITCH: With this switch in the "Patch" position, the D.V.M. is connected to the pre-patch panel. When the switch is in the "Sig Sel" position, the D.V.M. is connected to output of the component that is selected by the signal selector pushbuttons, with the V.T.V.M. switch in the Patch Panel position.

(4) $1000-\mathrm{v} / \mathrm{sec}-100-\mathrm{v} / \mathrm{sec}$ SWITCH: This switch determines the gain of the "Check" amplifier. In the $1000-v / \mathrm{sec}$ position the check amplifier has a gain of 0.1 and in the $100-\mathrm{v} / \mathrm{sec}$ position the amplifier has gain of unity.

(5) CHECK: The overload indicator shows when the check amplifier is overloaded. The amplifier is balanced by depressing the pushbutton and adjusting the balance control.

(6) VOLT'S SENSITIVITY: Selects the full scale voltage range of the V.T.V.M. The balance control located to the left of the volt's Sensitivity switch is for setting the electrical zero of the V.T.V.M.

(7) EXT. V.M.: The binding posts are used for connecting an external volt meter or an oscilloscope in parallel with the V.T.V.M. input.

(8) V.T.V.M. SELECTOR SWITCH: "Sig Sel" pushbutton connects V.T.V.M. to output of signal selector switch, and disconnects the D.V.M. if it is connected.

"Patch Panel" pushbutton connects the input of the V.T.V.M. to the pre-patch panel terminals and connects the D.V.M. to the sig sel switch, if the D.V.M. switch is in the sig sel position. Vib Drive pushbutton connects the output of the vib drive power supply to the V.T.V.M. The "+ $300 "$ pushbutton connects the output of the +300 -volt power supply to the V.T.V.M. The "+ $110 "$ pushbutton connects the output of the +110-volt power supply to the V.T.V.M. The "+ Ref." pushbutton connects the output of the +reference supply to the V.T.V.M. 
The "REF BAL" pushbutton connects the junction point of two precision resistors to $V_{0} T_{0} V_{0} M_{0}$ these resistors are connected in series between the +100 -volt and -100 -volt reference supplies.

The "Relay" pushbutton connects the output of the relay power supply to the V.T.V.M. The "-300" pushbutton connects the output of the -300 -volt power supply to the $V_{0} T \cdot V \cdot M$. The "-500" pushbutton connects the output of the -500 -volt power supply to the $V_{*} T_{*} V . M$.

(9) RATE TEST: The "Rate Test Potentiometer" adjusts the test voltage applied to the integrators during the dynamic test. The "Res. Check" pushbutton applies +100 volts to all the test resistors for testing.

The "Hold" pushbutton energizes all hold relays. The " $+100 \mathrm{v}$ and $-100 \mathrm{v}^{\prime \prime}$ switch connects the top of the rate test potentiometer to $+100 \mathrm{v}$ or $=100 \mathrm{v}$ or to the ground.

(10) OVERLOAD: The indicators show where the overload is occurring, either in the Ampl's or in the Servo"s. The "Auto Hold" switch, when thrown to the right position, causes the computer to be placed in the hold position when any overload occurs in the ampl's or the servo's and the computer will remain in the hold position until the control switch is turned to the Reset position. With this switch in the "Ampl Only" position, any overload amplifier will cause the computer to be placed in the hold position. In the center position, the overloads are indicated by the audio and the visual indicators, but the computer is not placed into the HOLD condition.

(11) SLAVE SEL。: Determines which machine will be slaved to this console or which one this machine will be slaved to.

(12) CONTROLS: The "Slave" position permits the control of the Pot-Set Reset and Hold relays by another computer. The "Rate Test" position allows the dynamic tests to be performed with the integrators. The "Pot-Set" position removes the input signals from the amplifiers by disconnecting the inputs at the summing point and grounding them. The grounding of the inputs permits the attenuators connected to the amplifier input networks to be set with normal load.

The "Re-set" position connects the inputs to the summing amplifiers and connects the "initial condition" voltage to the integrating capacitors.

The "Hold" position removes the "Initial Condition" inputs and all other inputs from the amplifiers. The capacitors remain charged to the "I.C." voltage. The "Operate" position starts the Integration by connecting all inputs to the amplifiers except the initial condition inputs.

The summing amplifiers operate in Reset, Hold and Operate positions. 
(13) START-STOP: This switch is the filament switch and turns the filam ment power on and off.

(14) PLATE-REF: This switch controls the application of the plate and the reference voltage. In the center position only the plate voltage is on. In the up position the plate voltage is on and the reference voltage is applied to the pre-patch panel. In the down position, both supplies are off. 


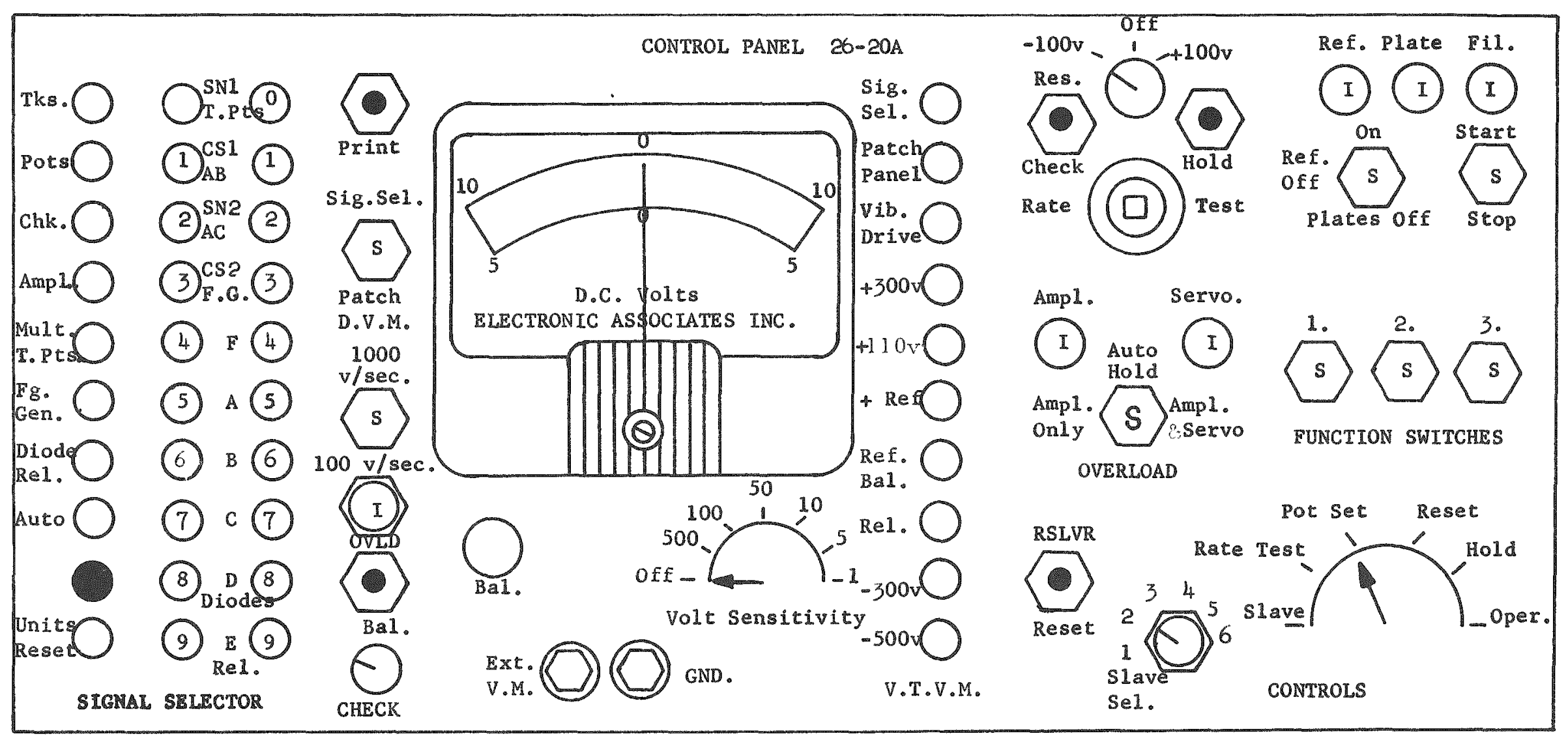

I - Indicator Bulb

S - Switch

Figure 8

CONTROL PANEL DIAGRAM 


\section{DIODE FUNCTION GENERATORS}

The five diode function generators available on each console are located in the servo rack just above the reference supplies. The diode function generators are used to produce electrical representations of arbitrary functions of the input variables. The generators may be used to approximate the curve of an arbitrary function with twenty segments or it may be used to approximate two curves of ten segments each by using the split operation switch. The controls on the D.F.G. are 1) a dual-channel switch for split operation of the unit, 2) a break-point control for each of the twenty segments, 3) a slope control for each segment, 4) a scale-factor control for the entire function, 5) a parallax control for the entire function, and 6) a balance control and overload indicators for the DC amplifiers.

The straight line segment approximations are generated by the use of biased diodes and their associated circuitry. The biased diodes operate with input signals between \pm 100 volts. The outputs of the biased diodes are compared and amplified by the stabilized DC amplifiers. The open loop gain of the DC amplifiers is above 20,000 and the proper input elements and feedback elements are incorporated to permit scale factors of $\frac{1}{2}, 1,2$, and 4. The parallax control provides a means of varying the DC level of the arbitrary function between \pm 100 volts.

The recommended method of setting up the diode function generator for operation is accomplished by using a variplotter inconjunction with an integrator.

The following diagram (Figure 9) shows the circuit arrangement for the set-up of the unit.

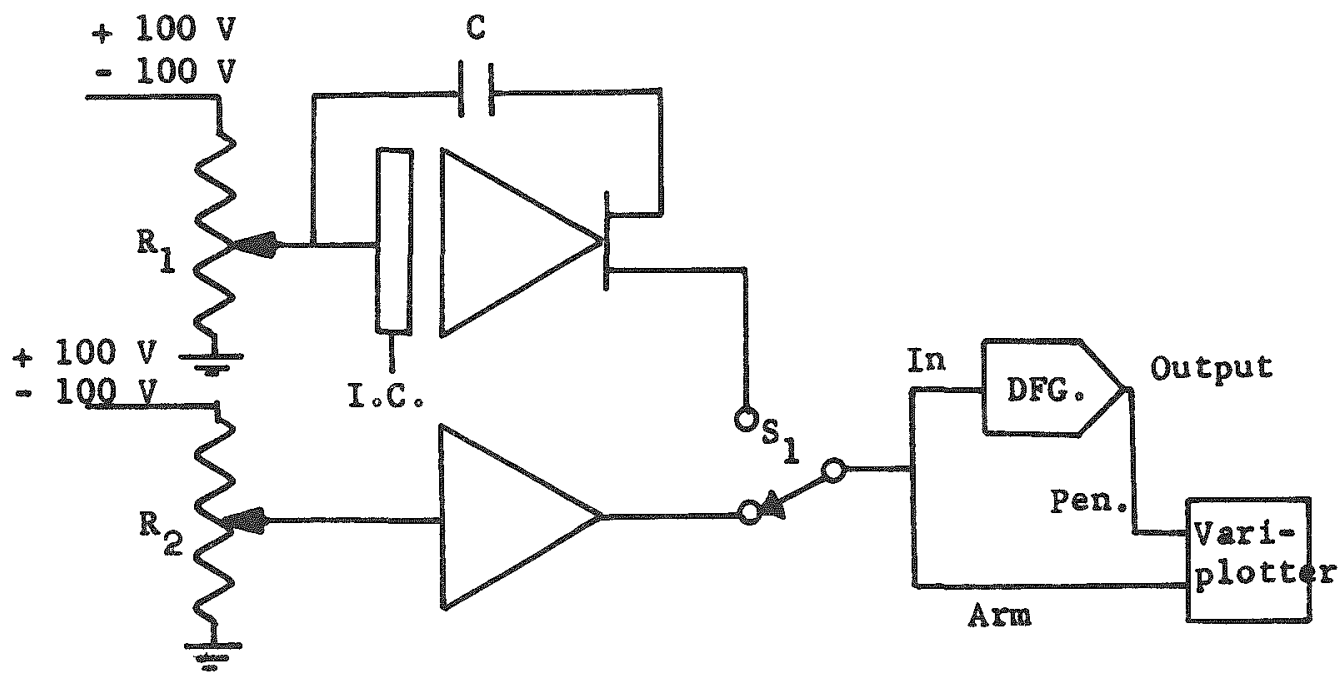

Figure 9

D.F.G. SET - UP CIRCUIT 
The following curve (Figure 10) shows the function that is being used as an example for the set-up procedure.

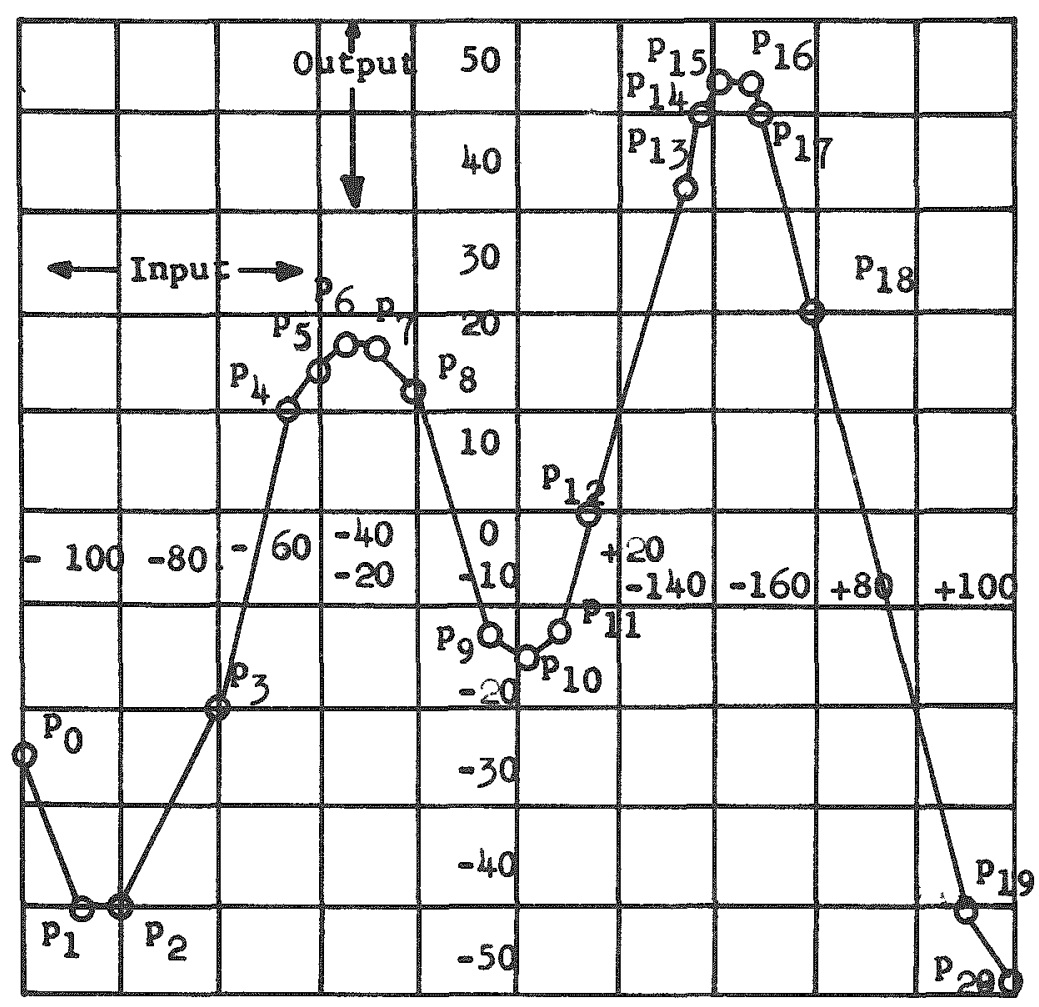

Figure 10

SAMPLE D.F.G. CURVE

The steps for the set-up procedure are listed below.

Step 1. Wire the circuit shown in Figure 9.

Place the curve of the desired function on the plotting surface of the variplotter.

Set the scale-factor control of the variplotter to correspond to the scale factor of the curve.

Set all of the break-point and slope controls of the D.F.G. to their counterclockwise position.

Use a dry pen in the variplotter so that the pen will be allowed to trace the curve in the down position, without marking the curve.

Step 2. Set the D.F.G. parallax control so that the pen is located at the starting point, $P_{0}$. The input voltage to the amplifier should be of opposite polarity to the input desired to the D.F.G., since there is a phase reversal in the D.F.G. amplifier.

Set the D.F.G. scale-factor control to 4.

Set the variplotter pen scale factor to 10 volts per inch.

Set the variplotter arm scale factor to 20 volts per inch.

Set the arm and pen parallax controls to zero. 
Step 3. Vary the position of the first break-point control until the pen just begins to move.

Step 4. Adjust the input voltage of the amplifier to a value which causes the pen to assume the $X$ position of $P_{1}$. This adjustment will change the $Y$ position but does not set it, it sets only the $X$ position.

Step 5. Adjust the position of the first slope control until the pen attains the $P_{1}$ position. This adjustment sets the $Y$ position. The first point has now been set.

Step 6. Vary the position of break-point control number 2 until the pen starts to move.

Step 7. Adjust the input voltage again until the pen comes to the $P_{Z} X$ position.

Step 8. Adjust the No. 2 slope control until the pen comes to the $P_{2}$ position. Point $\mathrm{P}_{2}$ has now been set.

Step 9. Proceed as above, setting in order for each point, the breakpoints, inputs, and slopes until all of the points have been set. The input voltage changes from minus to plus between the points $P_{g}$ and $P_{10}$. This indicates that the voltage at the upper terminal of $R_{2}$ will have to be changed from +100 to -100 volts when setting $P_{10}$.

Step 10. Switch $S_{\mathbb{1}}$ may now be set to connect the integrator to make a test run. By varying $R_{I}$, the speed in volts per unit time may be adjusted to suit the display or writing speed. After the entire curve has been set up and a satisfactory "dry run" has been made, then the wet run may be made to make a permanent record of the function.

The diagram, the example curve, and the procedure for setting up the diode function generator for operation have been taken from the P.A.C.E. Techniques engineering bulletin No. 16. 


\section{ELECTRONIC MULTIPLIERS}

The purpose of the electronic multiplier is to multiply or divide two rapidly changing variables by a third. It has the advantage over the servo multipliers in that it is capable of operating at much higher frequencies, since it is not limited by mechanical parts. There are five electronic multipliers in each console and they have their inputs, $A, B$ and $C$, and their outputs, $-A B / 100$ and $-A C / 100$, brought out on the lower right section of the pre-patch panel. The units can be used for division by changing the position of the switch labeled "MULT-DIVIDE" on the front panel. The outputs when the unit is being used for division are - $A 100 / B$ and - $A 100 / C$. Since there is an odd number of stages in the amplifiers, there is a sign inversion performed inside the unit.

The multiplication is accomplished by a process known as stabilized time division. This process utilizes a rectangular wave, where the width of the pulse is determined by the $A$ input and the height of the pulse is determined by the $B$ or $C$ input. The true average value of the resultant rectangular wave is proportional to the product of the two input variables, $A B$ or $A C$. The circuit associated with the electronic multiplier may be divided into three parts. Channel A is a pulse width circuit, channel $B$ is a pulse height circuit and channel $C$ is identical to channel $B$. Channel $A$ is the common multiplier and the channels $B$ and $C$ are the individual multiplicands.

Channel A provides two rectangular waves whose amplitudes are constant and equal but of opposite phase. The width of each of the pulses is directly proportional to the variable voltage fed to the input of channel A.

Channel B extracts the gate pulses from channel A and varies their amplitude directly proportional to the B input signal. The resulting rectangular waves are averaged in channel $B$ and yield an output voltage proportional to the combined effect of the pulse width and the pulse amplitude, or proportional to the $A$ and $B$ inputs. The operation of channel $C$ is the same as channel $B$.

The 10-kc generator or delay generator consists of a multivibrator, a diode-connected triode tube used as a limiter, a cathode follower, a phantastron delay tube, a squarer and a phase inverter, and a push-pull power output stage.

The multivibrator is a conventional free-running multivibrator, utilizing a twin-triode to generate a $10-\mathrm{kc}$ signal. The period of each cycle of operation is 100 microseconds. The circuit parameters are arranged so as to produce negative pulses of 10 -microsecond duration. The pulses are taken from the plate of the triode tube and applied to the phantastron delay tube. 
The purpose of the phantastron delay is to provide an output pulse whose amplitude is constant, but whose width is directly proportional to a negative control voltage obtained from the output of DC amplifier number 2. The phantastron delay circuit consists of a limiter, a cathode follower and a phantastron. The control voltage is coupled to the plate of the phantastron by the limiter tube. The limiter circuit is used for the purpose of preventing the control voltage source from loading the phantastron by disconnecting the control voltage as soon as the phantastron fires. The cathode follower is used for the purpose of decreasing the time required for the recovery of the plate voltage of the phantastron, thus producing a sharply defined output pulse. The duty cycle of the phantastron output pulse is defined as the ratio of the delay time to the time between successive input pulses. The output pulse takes the form of a series of rectangular waves.

The squarer is used to square off the extremities of the input signal and to provide an output which is a sharply defined rectangular wave. The other section of the squarer and phase inverter tube is used to provide a signal of the same amplitude but of opposite polarity to the output of the squarer. The plates of this tube provide an output voltage which is approximately of the same amplitude but of opposite polarity.

The phase inverter provides push-pull outputs to the power stages. When one of the output stages is operating on a positive pulse, the other stage will be operating on a negative pulse and rice versa. Each of these tubes produces a rectangular ware output of opposite polarity. The output of one stage of the power output stages is fed to one section of the switch tubes, and the output of the other stage is fed to other sections of the switch tubes

The DC amplifiers that are used consist of a twin-triode input stage, a pentode amplifier and a cascade output stage The gain of the cascade output stage is in excess of 40,000 . The balance control on the front panel of each amplifier adjusts the operational level of the grid of the pentode amplifier The overload indicators for the eight amplifiers indicate when the summing points of the circuits are nolonger at ground potential. After the amplifiexs have been manually balanced, the automatic balancing cir cuit prevents any drift ir, the amplifier.

In the division operation mode the magnitude and positive sign of one input control the height of a pulse, and the magnitude and sign of the other input control the duty cycle of the pulse. The pulse area is then proportional to the quotient of the two input variables.

There are two adjustments that must be made before the multiplier is put into operation if the results are to be accurate. These are the balancing of the DC amplifiers and nulling the bridge circuits. The amplifiers are balanced by depressing the pushbutton of each amplifier and 
adjusting the balance control until the neon indicator is extinguished for each amplifier. The null adjustments are accomplished by adjusting 8 trimming potentiometers, 4 in the $B$ channel and 4 in the $C$ channel. These trimming potentiometers have the purpose of compensation of small changes in resistor values in order to provide the required ratios for the various bridge circuits in each of these channels. When performing this adjustment the null selector switch is turned to position number 1 and trimmer number 1 is adjusted for a null on the null meter on the control panel. In position number 2 , trimmer number 2 is adjusted for minimum deflection on the null meter while changing the reference switch from normal to reverse. Position number 3 is adjusted for a null on the null meter while changing the reference switch from normal to reverse. In position number 4 , trimmer number 4 is adjusted for minimum deflection in the same manner. Positions 5 through 8 are adjusted in the same manner for the C channel. The null selector switch should be turned back to the OP position, attempting to null the next multiplier unit.

The null procedure for division is accomplished by applying zero voltage to the $A$ input, 1.0 volt to the $B$ input and adjusting the number 1 null position until there is less than 1.0 -volt output from channel $B$. The number 2 null position is adjusted by applying 20 volts to the $A$ input, 100 volts to the $B$ input, and adjusting trimmer number 2 until the output from the $B$ channel is less than 0.025 volt. The other adjustments are adjusted by the multiplier adjustment procedures.

The C channel remote switch on the front panel of the multiplier unit is for the purpose of slaving the $C$ channel of each multiplier to the A channel of one multiplier. The units are so wired that, when the $C$ channel NORMAL-REMOTE switch is placed in the REMOTE position, the $C$ channel of that unit is slaved to the $A$ channel of the unit directly below it. This feature enables a variety of input variables to be multiplied by the group. The slaving of the units for division is accomplished by placing the $A B$ output of one multiplier into the $A$ input of another multiplier and placing the $C$ channel NORMAL-REMOTE in the normal position.

In addition to the power supplies that are used for the other amplifiers throughout the computer, the multiplier rack utilizes a Stabilizer unit. The purpose of the stabilizer is to hold the -300 -volt output steady with the +100 -volt reference. 


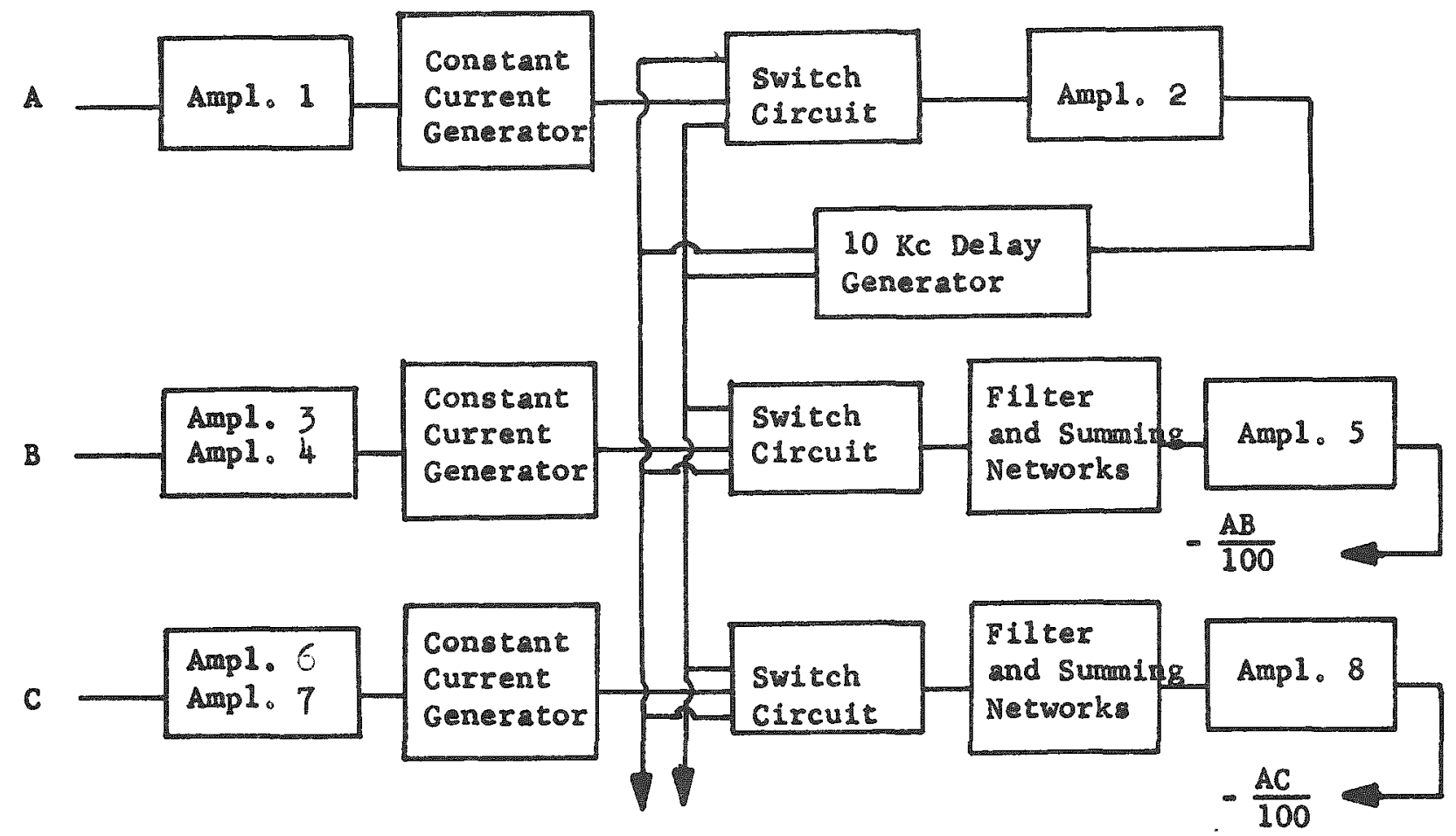

Figure 11

BLOCK DIAGRAM OF ELECTRONIC MULTIPLIER
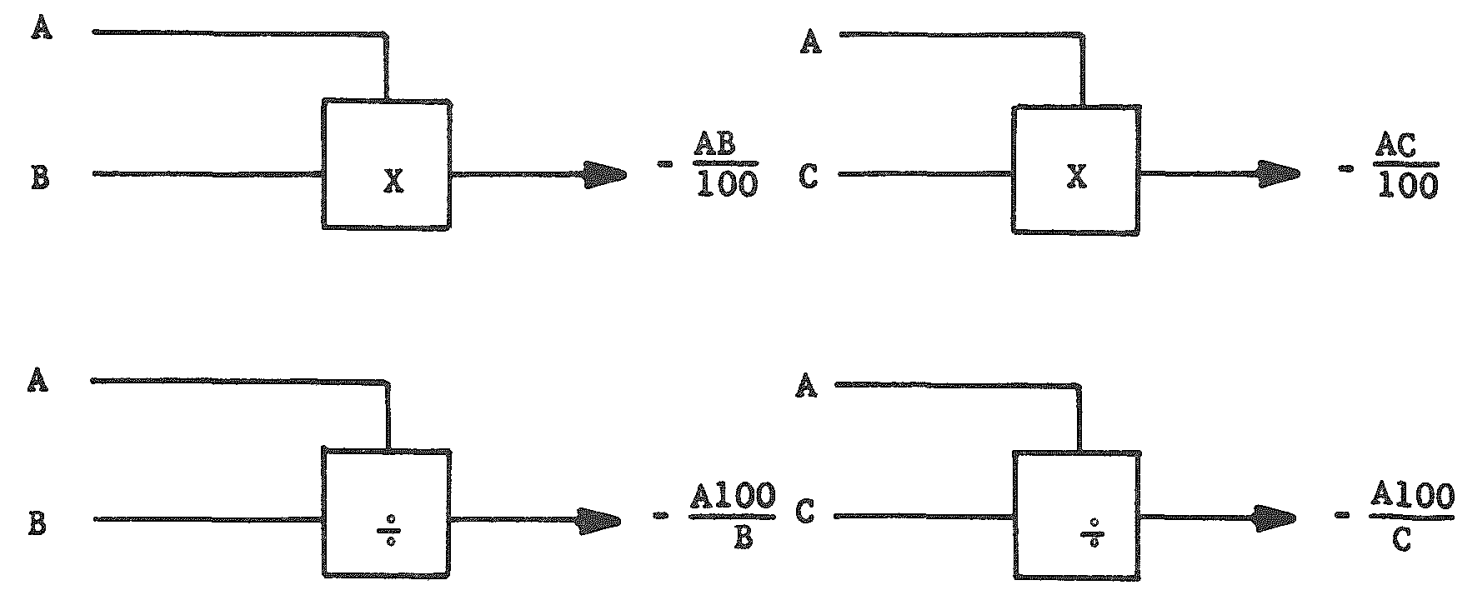

Figure 12

ELECTRONIC MULTIPLIER SYMBOLS 


\section{NET WORKS}

The operational amplifiers are classed into two groups: the combination amplifier and the summing amplifier. The dual-purpose operation is made possible by the use of two different types of relay networks.

The combination amplifiers (numbers 1 through 28) can be used for integrating, summing and inverting, depending upon the location of the bottle plug at the top of the amplifier position on the pre-patch panel. When the amplifier is to be used as an integrator, the bottle plug is inserted into the lower four terminals and the letters "INT" will show directly above the bottle plug. The bottle plug connects the summing point of the amplifier position to the "H" terminal, which connects the common point of the input resistors to the input side of the feedback capacitor through the hold relay. The output side of the feedback capacitor is permanently connected to the output of the amplifier. Then the input side of the feedback capacitor is connected by the bottle plug to the feedback resistor, which is permanently connected to the grid of the amplifier. The $1.0-m e g o h m$ feedback resistor is now connected between the summing point and the grid of the amplifier.

There are three relays used in the combination amplifier circuit. The "Pot-Set" relay connects the input resistors to the grid of the amplifier when it is de-energized. When the pot-set relay is energized it connects the common point of the input resistors to high-quality ground. The "Re-Set" relay connects the input side of the feedback capacitor to a resistor network between the output of the amplifier and the "Initial Condition" terminal on the pre-patch panel. The "Hold" relay permits the input of the feedback capacitor and the grid of the amplifier to be disconnected from the summing point of the input resistors. Operating the hold relay causes the feedback capacitor to remain charged and the amplifier output remains constant at the ralue which it had when the hold relay was energized.

The "Initial Condition" terminal permits a problem to be started with any desired voltage at the output of the integrators. When the desired voltage is patched into the "I.C." terminal on the pre-patch panel, the feedback capacitor will be charged to the magnitude of this voltage and the output of the amplifier will be of the same magnitude but of opposite polarity.

The combination amplifier networks have provisions for seven inputs. Three of the inputs are connected to 1.0 -megohm resistors for a gain of one. Two of the inputs are connected to $0.2-\mathrm{megohm}$ resistors for a gain of five, and the last two inputs are connected to 0.1 -megohm resistors for a gain of ten. The diagram for the combination amplifier relay network is shown in Figure 13 


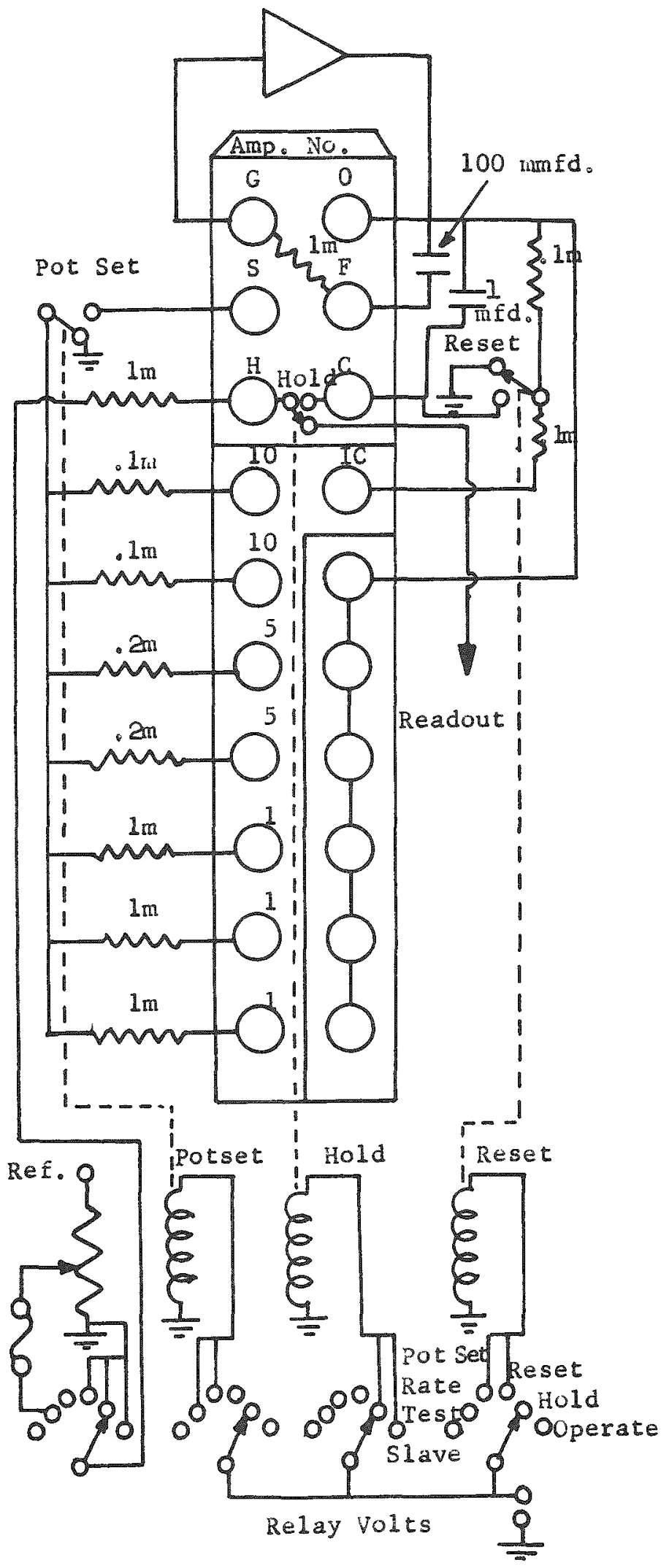

Figure 13

COMBINATION AMPLIFIER NETWORK 
The remaining amplifiers (numbers 29 through 56 ) are used as summing or inverting amplifiers only. The operation of the combination amplifier, with the bottle plug inserted into the upper four terminals, is the same as the operation of the summing amplifier, with the exception of the number of inputs. The summing amplifier has only five inputs. One input is for a gain of ten, one input is for a gain of five and the other three inputs are for gains of one each.

There are four terminals brought out to the pre-patch panel on the last 28 amplifiers. They are (1) the grid terminal, (2) the summing point of the input resistors, (3) the amplifier output and (4) the feedback resistor terminal. When the bottle plug is inserted into one of these positions it connects the output of the amplifier to the feedback resistor, which is connected on the other terminal to the grid of the amplifier. Operation of the summing amplifier involves only one relay, that is, the "Pot-Set" relay. This relay connects the summing junction of the input resistors to ground when energized and connects the summing junction to the grid of the amplifier when it is de-energized. The input resistor networks, and the relay networks and the feedback capacitors are located in a temperaturecontrolled oven directly behind the pre-patch panel. The circuit for the summing amplifier relay network is shown in Figure 14 . The symbols for the different types of amplifiers with their inputs is shown in Figure 15. 
ㄱำ $2 \operatorname{xn} 8 \div A$

XIOMZ ON

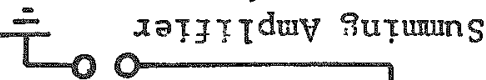

'Si วxnรt]

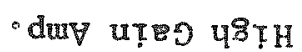

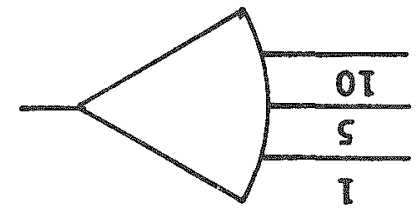

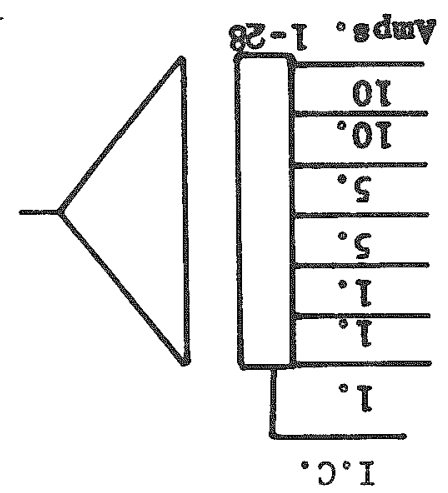
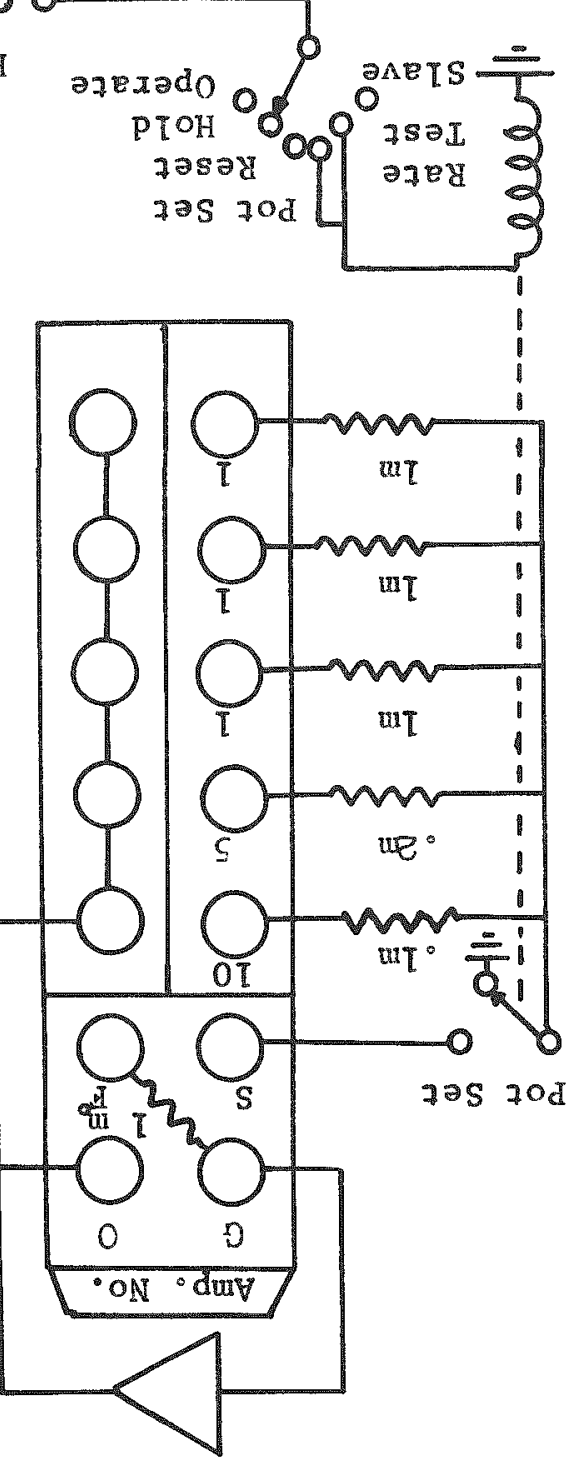


\section{NOISE GENERATOR}

The electronic noise generator produces a stable and reliable source of random noise which is used in the study of control systems and missile systems. The generator, located below the console desk on the computer, has an output in the frequency range from zero to 35 cycles per second with a Gaussian or normal distribution of amplitudes. There is an automatic level control to stabilize the output against the computer reference voltages. The output of the generator is monitored on a silicone fluid-damped meter. This fluid provides a smoothing time of several. minutes. The maximum output level is 15 volts r.m.s. and it may be decreased by an attenuator.

The primary source of noise in the generator is the $2 \mathrm{D} 21$ gas thyratron tube. The output of this gas tube is a band of noise extending from $30 \mathrm{cps}$ to $3 \mathrm{kc}$. The level of the output of the gas tube is affected by the heater potential and envelope temperature. The $30 \mathrm{cps}$ to $3 \mathrm{kc}$ noise output passes through a regulator circuit, and the noise output of the regulator circuit has a constant average amplitude in the same frequency range. The noise output from the regulator circuit is filtered through a 400-cycle band pass filter. At this point the narrow band of noise is amplified and phase detected by the 400-cycle chopper, and then passes through a low pass filter which removes the high-frequency components. The output is now uniform in the frequency range of from zero to $35 \mathrm{cps}$. The block diagram of the noise generator is shown in Figure 16.

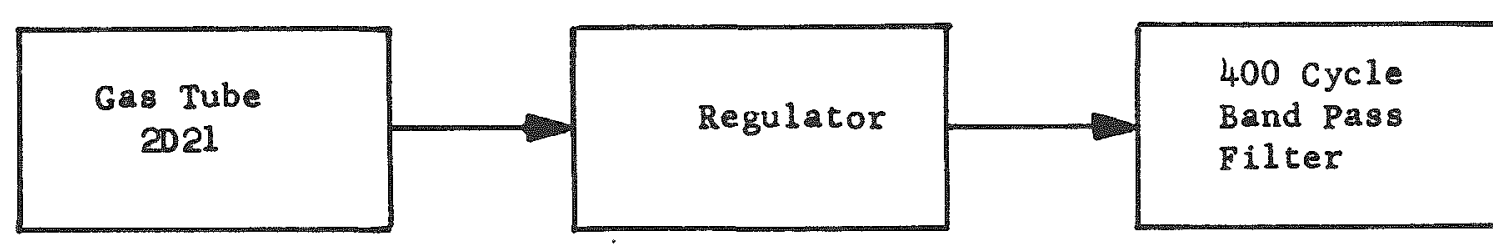

400 cycle

Amplifier Chopper and

Filter

Output

Figure 16

BLOCK DIAGRAM OF NOISE GENERATOR 
The controls and indicators on the front panel of the noise generator include a bias control that adjusts the noise input to the variable gain pentode in the regulator circuit. The bias, indicated on the bias meter, should be kept in the range from 15 to 30 volts. The output control adjusts the noise output of the regulator system to control the output of the generator. The output, indicated on the silicone-damped meter, should always be adjusted for a reading of 25.0 .

In the event the noise generator fails, the square wave output of the chopper is brought out to a jack on the front panel for checking. Upon connecting this jack to an oscilloscope and turning the OFF-TEST-OPERATE switch to the test position, the output wave form may be viewed. This waveform should be clean, symmetrical and have a peak-to-peak amplitude of approximately 13 volts. 


\section{OPERATIONAL AMPLIFIER}

The operational amplifier is basically two amplifiers. One is a DC amplifier that has a gain of approximately $2 \times 10^{5}$; the other is an $A C$ amplifier, with a gain of $3 \times 10^{3}$, that is used for the purpose of automatically correcting for any drift in the DC amplifier. The combined gain of these two sections results in an overall amplifier gain of $6 \times 10^{8}$. The normal operating range of the amplifier is $\$ 100$ volts into a load of $7500 \mathrm{ohms}$. If the load on the output of the amplifier is reduced, then the operating range can be extended to between \pm 100 rolts and \pm 200 rolts. The noise output of the amplifier should be less than 10 millivolts peak. This noise is caused by microphonic tubes or excessive 60 cycle in the output which is caused by heater to cathode leakages near the input. After the amplifiers have been balanced the normal long-time offset is less than 75 millivolts for a 24 -hour period. The frequency response of the amplifier is from 0 to the kilocycle range.

The DC amplifier section consists of a twin-triode input stage, using a 5691 -type tube, followed by two pentode amplifier stages, using either a 5963 or a $6 \mathrm{SJ} 7$ in the first stage and a $6 \mathrm{AC} 7$ in the second stage. The pentode amplifiers are followed by a triode-connected cathode follower output stage that uses a 5881 tube.

The AC stabilizer amplifier consists of a 94-cycle synchronous vibrator followed by two stages of amplification, using the type 5963 or $6 S J 7$ tubes. The AC circuit assumes control of the balancing of the DC amplifier after it has been balanced manually. Eigure 17 is a block diagram of the amplifier.

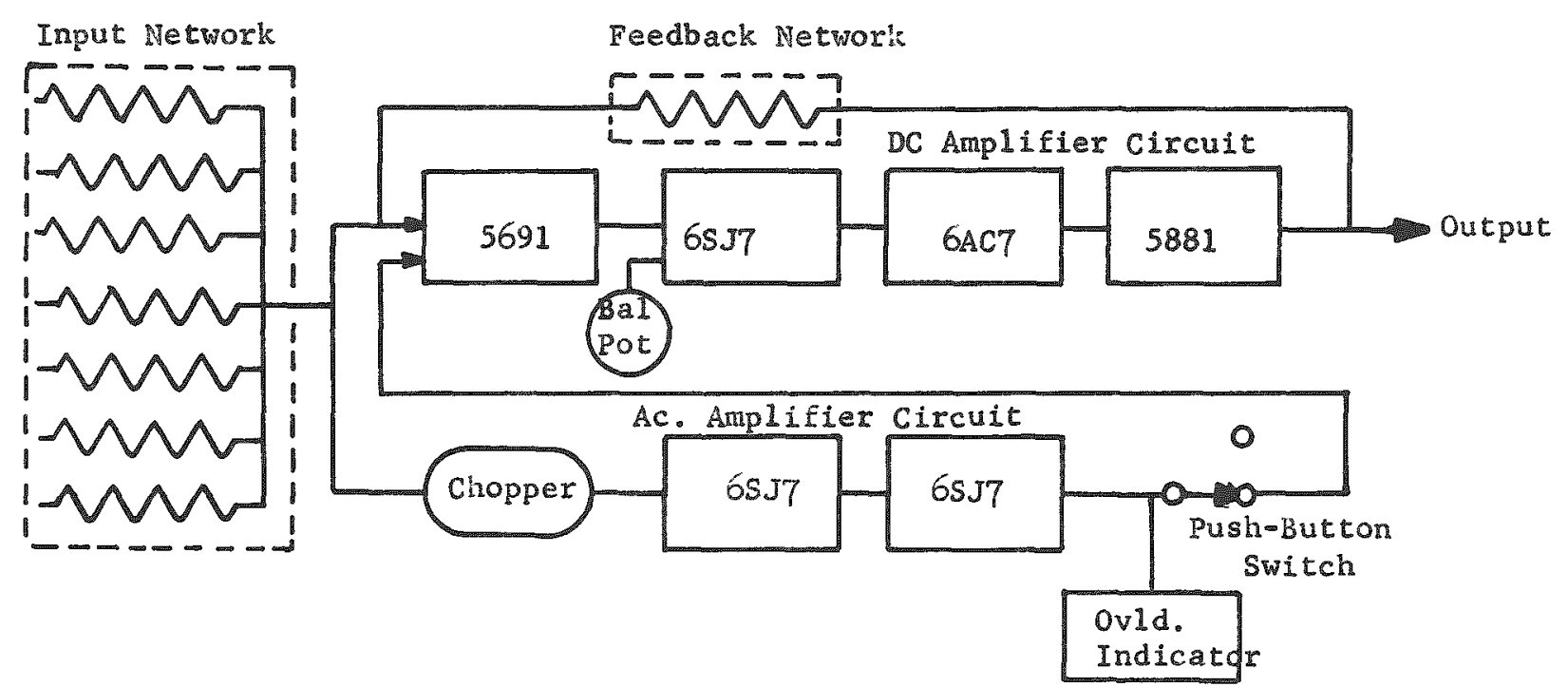

Eigure 17

AMPLIFIER BLOCK DIAGRAM 
In order for the amplifier to operate properly and to give accurate results there must not be any drift, or when there is no input there should be no output. The drift has been reduced by reducing the grid current of the input stage. Drift can occur anywhere in the amplifier, but it is more serious in the first or input stage, since it would be amplified by three stages of amplification and then would appear as a large quantity at the output of the amplifier.

On the front panel of the amplifier chassis there is an overload indicator, a balance control and a balance pushbutton. The neon indicator bulb indicates when the input terminal is no longer at ground potential, when the AC amplifier is short circuited, and when the amplifier is operating beyond its normal voltage range. The balance control and the pushbutton are for balancing the DC amplifier. This is done by depressing the pushbutton, which grounds the output of the AC amplifier, and adjusting the balance control until the neon bulb is extinguished. This control adjusts the operational level of the grid of the first pentode amplifier in the DC section.

In operation, the feedback element determines if the amplifier is to be used as a summer or an integrator. If the feedback element is a resistor, then the amplifier is a summer. The configuration of the summing amplifier is shown in Figure 18. The step-by-step derivation of the transfer function is made by assuming that the input grid current is zero and the drift and offset is zero. The output is 180 degrees out of phase with the input.

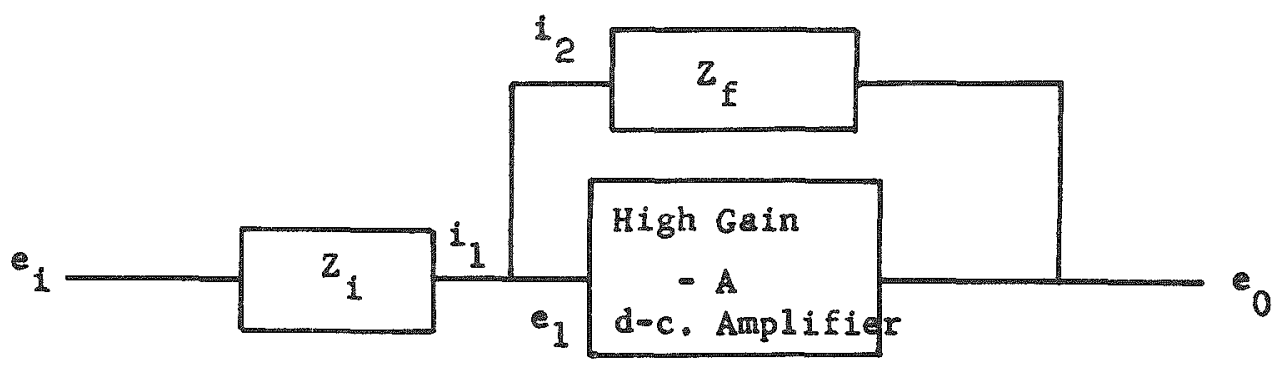

Figure 18

SUMMING AMPLIFIER
1. $\dot{i}_{1}=i_{2}$
4. $e_{0}=-A e_{1}$
7. $\frac{e_{0}}{e_{i}}=-\frac{A Z f}{Z_{f}+Z_{i}+A Z_{i}}$
2. $\dot{i}_{1}=\frac{e_{i}-e_{i}}{Z_{i}}$
5. $\frac{e_{i}-e_{1}}{Z_{i}}=\frac{e_{1}-e_{0}}{Z_{f}}$
8. $\frac{e_{0}}{e_{i}}=-\frac{Z_{f}}{Z_{i}}$
3. $i_{2}=\frac{e_{1}-e_{0}}{z_{f}}$
6. $\frac{e_{i}+\frac{e_{0}}{A}}{Z_{i}}=-\frac{\frac{e_{0}}{A}+e_{0}}{Z_{f}}$ 
The operational amplifier is changed from a summer to an integrator by changing the feedback element from a resistor to a capacitor. The input to the amplifier is still a resistor. The configuration for the integrator is shown in Figure 19.

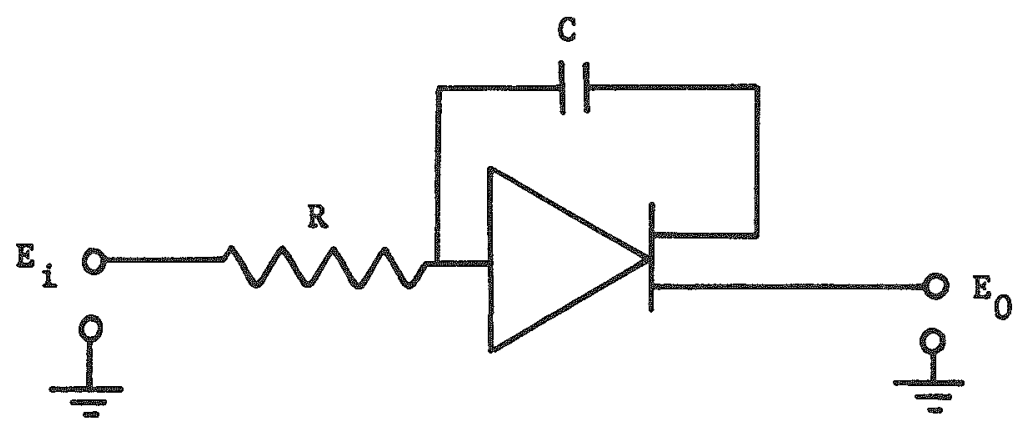

Figure 19

INTEGRATING AMPLIFIER

For integration the feedback element is a capacitor and the impedance is

$$
\begin{aligned}
& \frac{1}{j \omega C} \quad \frac{e_{0}}{e_{i}}=-\frac{Z_{f}}{Z_{i}} \\
& \frac{e_{0}}{e_{i}}=-\frac{1 / j \omega C}{R}=\frac{1}{j \omega C \cdot R} \\
& C=1 \times 10^{-6} \mathrm{Farads} \quad R=1 \times 10^{6} \mathrm{ohms} \\
& \frac{e_{0}}{e_{i}}=-\frac{1}{10^{6} \times 10^{-6} j \omega}=\frac{1}{j \omega} \\
& \frac{e_{0}}{e_{i}}=-\int e_{i} d t
\end{aligned}
$$




\section{OVERLOAD ALARM SYSTEM}

The overload alarm system is a very important feature of the computer, since it notifies the operator immediately when the amplifiers or the servos or any other components exceed their maximum operating ranges. The system also notifies the operator whenever there are any failures in the computer power supplies or components.

The system employed in this computer is a dual-channel system, with one channel for the servo units and one channel for the amplifiers. The indicators for the overloads are neon bulbs on the control panel, one for the servo units and one for the amplifiers, and a neon bulb located on the front of each individual unit. The other indicator is an audible alarm which operates when any unit is overloaded.

The only controls for the overload system is a volume control and a tone control for the audible alarm. The volume control and the speaker are located under the computer desk on the left side. The tone control is located on the overload chassis in the rear of the computer.

The overload alarm system provides the computer with an automatic hold feature, which means that any overload will cause the computer to be switched automatically to the "Hold" condition and remain there until it is switched manually to the "Re-Set" condition. The "AutoHold" switch is located above the neon bulb overload indicators on the control panel. When this switch is placed in the servo position, any over= load in the servo units will cause the auto-hold circuits to be energized and switch the machine automatically to hold; with the switch in the amplifier position, any overload in the amplifiers will cause the auto-hold circuits to be energized. If the switch is in the center position, the overloads will be indicated by the visual and the audible indicators, but the computer will not be switched into the hold condition. 
The "Pot Padder" units are used in conjunction with one of the tapped cups of a servo multiplier to generate arbitrary nonlinear functions. The tapped cups of the servo multipliers have scventeen individual taps. The pot padder panel provides facilities for setting up 17 predetermined voltages in accordance with a plotted curve. These voltages are applied to the taps of a servo multiplier for the purpose of function generation. The function generation circuit is shown in Figure 20.
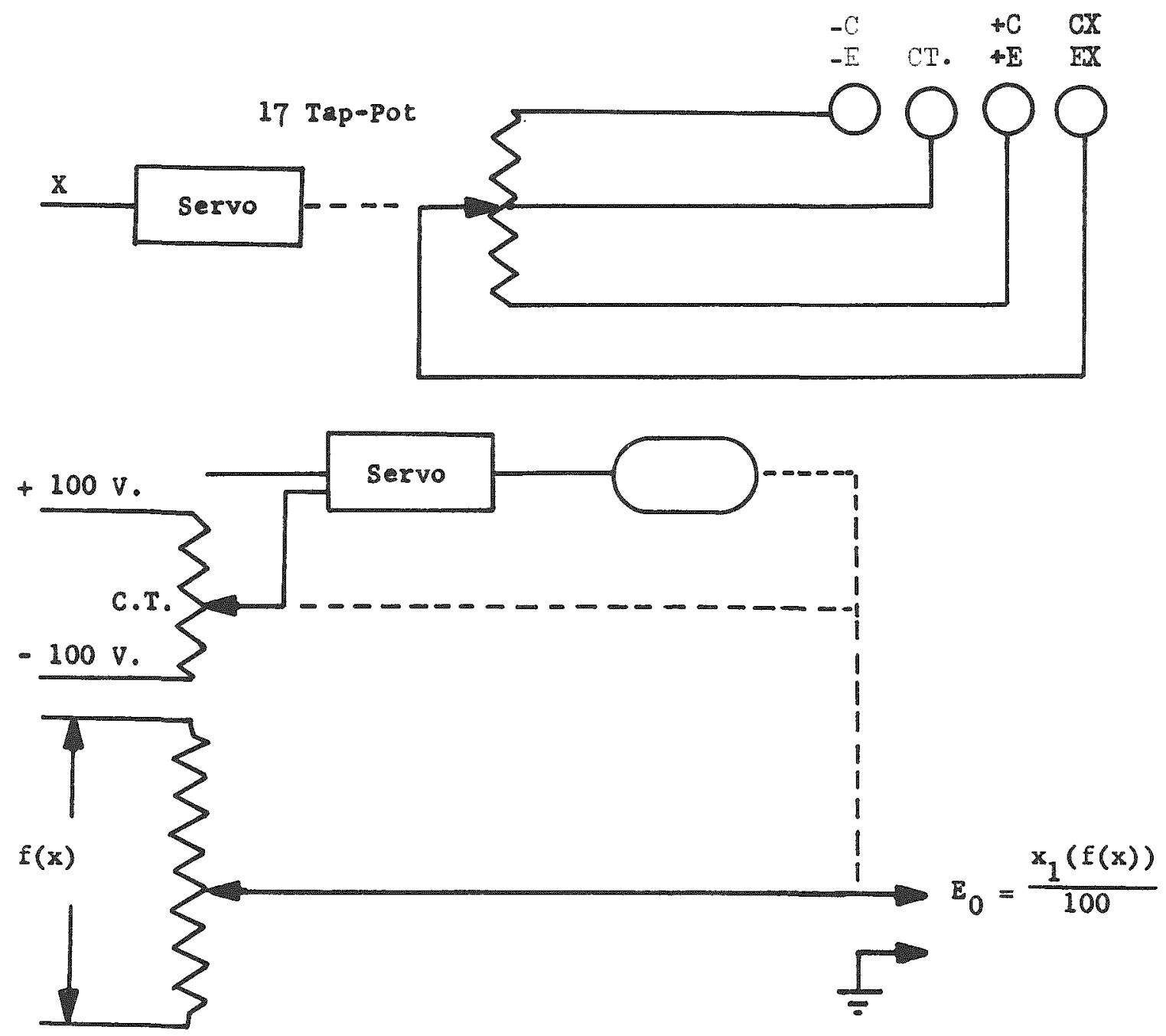

Figure 20

FUNCTION GENERATION CIRCUIT 
The controls that are used in setting up the pot padders are as follows:

1) The slope polarity selector switches, Sl through Sl 7, are the upper row of knobs that supply either open circuit, +100 -volt reference, ground, or - 100-volt reference to the upper terminals of potentiometers $R l$ through R17.

2) The slope control potentiometers, R1 through RI7, are the lower row of knobs that provides the desired tap voltages for the multiplier potentiometers.

3) The REF-PB switch, switch 18, in the REF position supplies the 100 volts and -100 volts to the slope polarity selector switches for application to the potentiometers.

In operation, the plus and minus reference voltages are supplied to each of the slope control potentiometers by means of the slope polarity selector switches. The slope control potentiometers are set under load and are set to the actual potential as plotted on the desired curve.

If the REF-PB switch is set to the $P B$ position, then the input will be a variable that can be patched in from the computer pre-patch panel. The resultant output will be the product of the plotted curve and the variable.

The set-up procedure for the pot padder units is outlined in the following 8 steps. The function that is being used as a sample for the set-up procedure is shown in Figure 21.

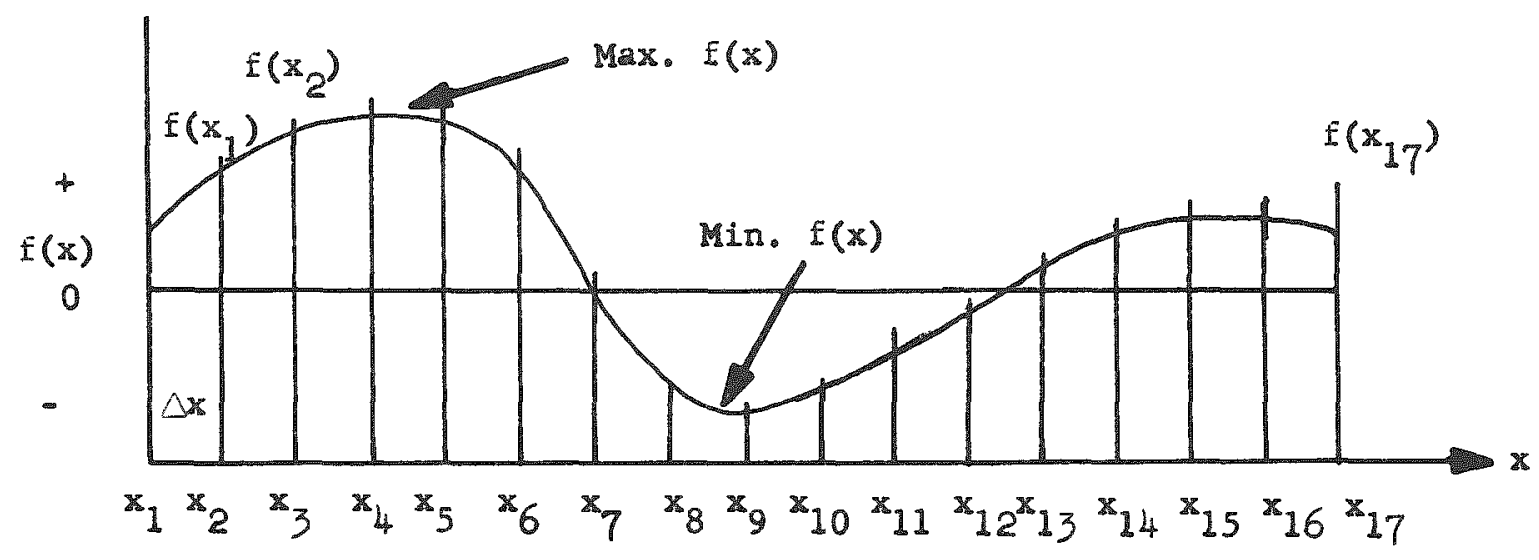

Figure 21

SAMPLE CURVE FOR POT PADDER SET-UP 
Step 1. Connect the Pot Padder unit to the desired servo cup with a rear cable. Connect the padder unit to the Pot Padder and D.F.G.control panel, a drawing of this panel is shown in Figure 22. The panel switch marked FUNCTION should be in the pot pad position. The D.V.M. is connected to the panel by selecting Function Generator No. 7 on the signal selector. Place the voltage switch on Pots rather than Taps.

Step 2. Draw a graph of the function, such as the one shown in Figure 21 .

Step 3. Set the load selector switch for the value of the load that the function will work into.

Step 4. Set the 17 reference potentiometers and reference selectors to values corresponding to the function.

A. Rotate the Pot Selector to the appropriate pot.

B. Supply voltage ( $\$ 100$ volts) to this pot from the corresponding switch on the control panel.

C. Place the readout selector switch in set-up Ampl position and read the output voltage on the D.V.M.

Step 5. Rotate all slope polarity selector switches and slope pots to the off position (counter clockwise).

Step 6. Set the REF-PB switch,

A. to REF position if the minimum $F(x)$ is approximately

-100 volts and the maximum $F(x)$ is approximately +100 volts.

$B$. to $P B$ position if the voltages for $F(x)$ must be patched in on the pre-patch panel. This is determined by the minimum and maximum amplitudes of the function that is being generated.

Step 7. Press the Pot Pad null switch; this is a spring-loaded switch.

A. If the needle on the null meter deflects to the right side, then set the polarity selector switch to + position.

B. If the needle deflects to the left, then set the polarity selector switch to the - position.

C. After setting the slope polarity switch, rotate the slope pot until there is a null on the meter.

Step 8. Release the Pot Pad null selector switch and then continue with the same procedure through all seventeen positions. 
After the set-up is completed, the Pot Selector switch should be set to position 18 for operation, and the cable to the control panel should be removed. The null selector switch should not be pressed after the set-up has been completed, since such an action is likely to cause the fuses on the arms of the pots to be blown.

The servo cups that are being used have a current limitation; therefore the voltage difference between two adjacent taps is limited to a maximum of 25 volts. It may be necessary to reduce the overall scale of the curve inorder to make the voltage between the taps fall into this range. The maximum $f(x)$ must be less than the positive voltage applied to the servo cup, and the minimum $f(x)$ must be less negative than the negative voltage applied to the servo cup.

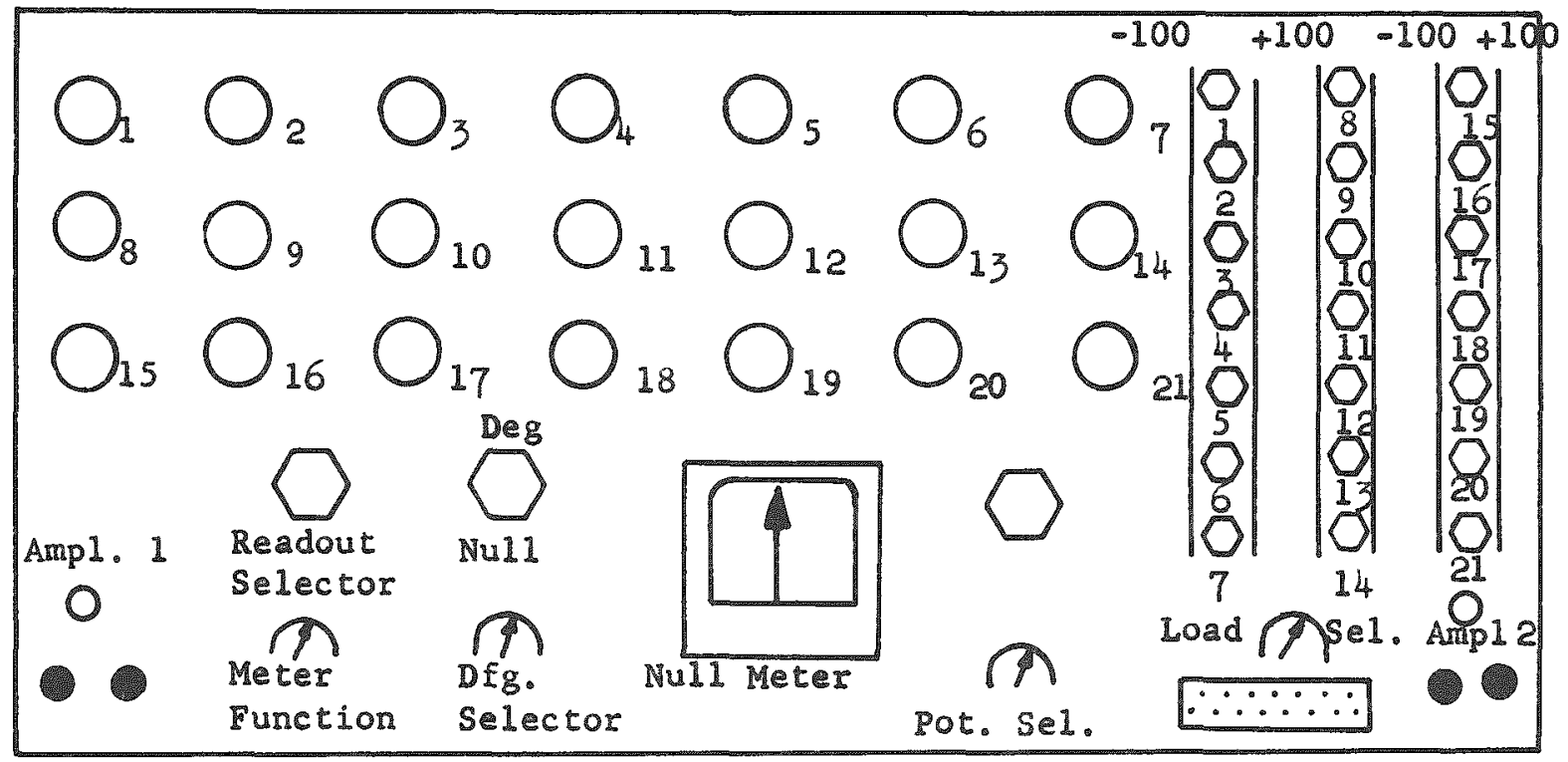

Figure 22

D.F.G. and Pot Padder Control Panel 


\section{POWER SUPPLIES}

The reference voltage power supplies are used to supply the computer with the \pm 100 volts for references with the signal voltages. The reference voltages are obtained by multiplying the 1.0192 volts of a standard cell to -100 volts. The +100 volts is obtained by inverting the -100 volts. The output stage of the amplifier contains nine 5881 tubes in parallel to allow a current drain on the supply of up to 1.0 ampere. The reference amplifiers are drift-corrected high-gain amplifiers, with a stabilizing circuit used to prevent drift due to a change in the load. The amplifiers contain an overload indicator and a balance control. The amplifiers should be balanced periodically and before starting a problem. In addition to the reference supplies being used for the signal voltage references they are used for potentiometer voltages for initial conditions, for references voltages for the follow-up pot of the servo multipliers, and the outputs of both the plus and minus supplies are brought out to the pre-patch panel for various other uses. Associated with the reference supply circuits there is an External-Internal switch. When this switch is in the Internal position the reference voltage is applied to the computer in the manner describedabove; when this switch is in the External position the reference voltage is connected into the computer from another source. The Internal and External connections of the reference supplies are shown in Figure 23.

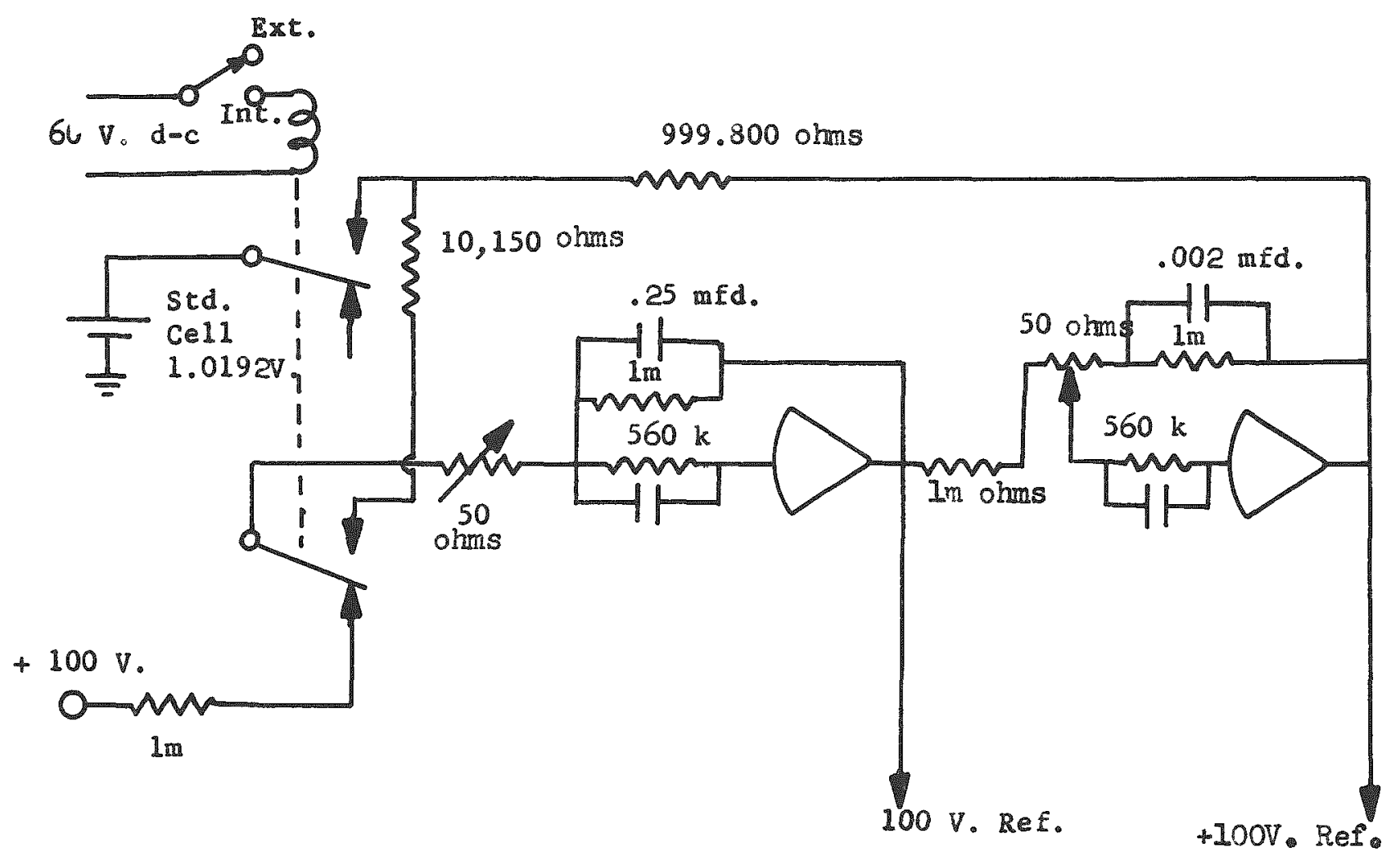

Figure 23 
The regulated power supplies required are the $\pm 300-$ volt $D C$ supplies to supply the plate voltage for the amplifiers and the 6.3-volt AC filament voltage for the electron tubes. These supplies have a regulation of $0.1 \%$ from no load to full load and a ripple voltage of less than two millivolts.

The -500 volts DC is obtained by combining the output of the -300 -volt supply and the inverted output of the +200 volt supply. In adjusting the -500 -volts, it is necessary to adjust the -300 -volt supply and then monitor the -500-volt supply and adjust the 200-volt supply.

The vibrator drive unit consists of a low-frequency oscillator, a para-phase amplifier and a push-pull output stage, with transformer output coupling. The output is a 94-cycle sinusoidal voltage with an amplitude range of from 0 to 20 volts. The vibrator drive unit is used to drive the synchronous converters or vibrators that are used to stabilize the DC amplifiers throughout the computer.

The electronic multiplier uses, in addition to the regulated power supplies, a stabilizer unit. This unit is used to stabilize the output of the -300 -volt power supply with the +100 -volt reference supply in order to prevent any drift in the electronic multiplier units.

The regulated power supplies must be checked periodically and ad. justed whenever necessary. They should always be checked before a problem is started on the computer. The outputs of all the supplies are brought out to a jack on the front panel of the cabinet in which they are located. The screwdriver adjustments of the supplies are located on the front panel of the supply chassis. The controls for adjusting the +110 -volt supply and the +200-volt supply for the electronic multiplier and the servo racks are located in the rear of their respective racks. 


\section{SERVO MULTIPLIERS}

The servo multipliers used in this installation are of two different types: 1) the model 16-7M uses a ten-turn potentiometer and 2) the model 16-7N uses a one-turn potentiometer The multipliers are located in the main console rack just above the control panel and in the upper left section of the servo rack.

The servo multiplier circuit is essentially a motor-driven potentiometer, a gear train and the associated servo amplifiers. The control inputs that are fed into the servo amplifier are the common varable signal and the feedback voltage. The common variable input voltage represents the magnitude of the common variable by which the other five variables are to be multiplied. The feedback is that voltage appearing on the wiper of the multiplying potentiometer. These inputs are applied, one to each end of an input network, and the voltage across this network is equal to the dif ference of the two inputs. At this network, the voltage proportional to the system error is generated. The DC exror voltage is now converted to a 60-cycle AC wave by means of a mechanical chopper The output of the chopper is applied to both ends of the primary of the input transformer and the primary center -tap is connected to the common variable input lead, which results in an input proportional to the difference between the signal input voltage and the voltage for the wiper of the multiplying potentiometer.

The output signal of the input transformer is a square wave with its amplitude proportional to the DC error signal level and a phase that reverses when the DC error signal changes polarity. The output of the trans former is applied to two successive stages of amplification, then to a phase splitter that is used to obtain balanced drive for the push-pull output stage. The output signal is then applied across an output transformer and taken from across the output transformer directly to the servo motor.

The gain of the servo, which is a screwdriver adjustment on the front panel, controls the amount of signal being applied to the second amplifier stage.

The damping control adjusts the degree of damping on the follow-up system by adjusting the amount of voltage that is being fed back to the input network

The electrical stop prevents any overtravel of the servo system when the signal voltages are equal in amplitude to the reference voltages that are applied. The electrical stop switch permits the removal of the electrical stop feature from cup No. 1 when desired. 
The overload indicator on the front panel is connected between highquality ground and the secondary of the output transformer. An overload is also indicated on the control panel. The overload indicator is energized when the tracking error exceeds 1.5 volts.

The potentiometers in the servos are ganged and driven by the servo motor. The multiplying pot, called the "Follow-Up," is normally connected with a constant voltage across it. The arm of the follow-up pot is brought out to the pre-patch panel for the purpose of connecting the external load resistor. The arms of the other pots, A, B, C, D, and $E$, are brought to the pre-patch panel for use in the problem circuit. The arms of all of the pots are fused with a $\frac{1}{32}$-ampere fuse for protection of the pots. The linearity tolerances of the single-turn pots are less than $0.24 \%$ maximum. The linearity tolerances of the ten-turn pots are less than $0.25 \%$ maximum. The schematic diagram of the multiplier pots is shown in Figure 24.

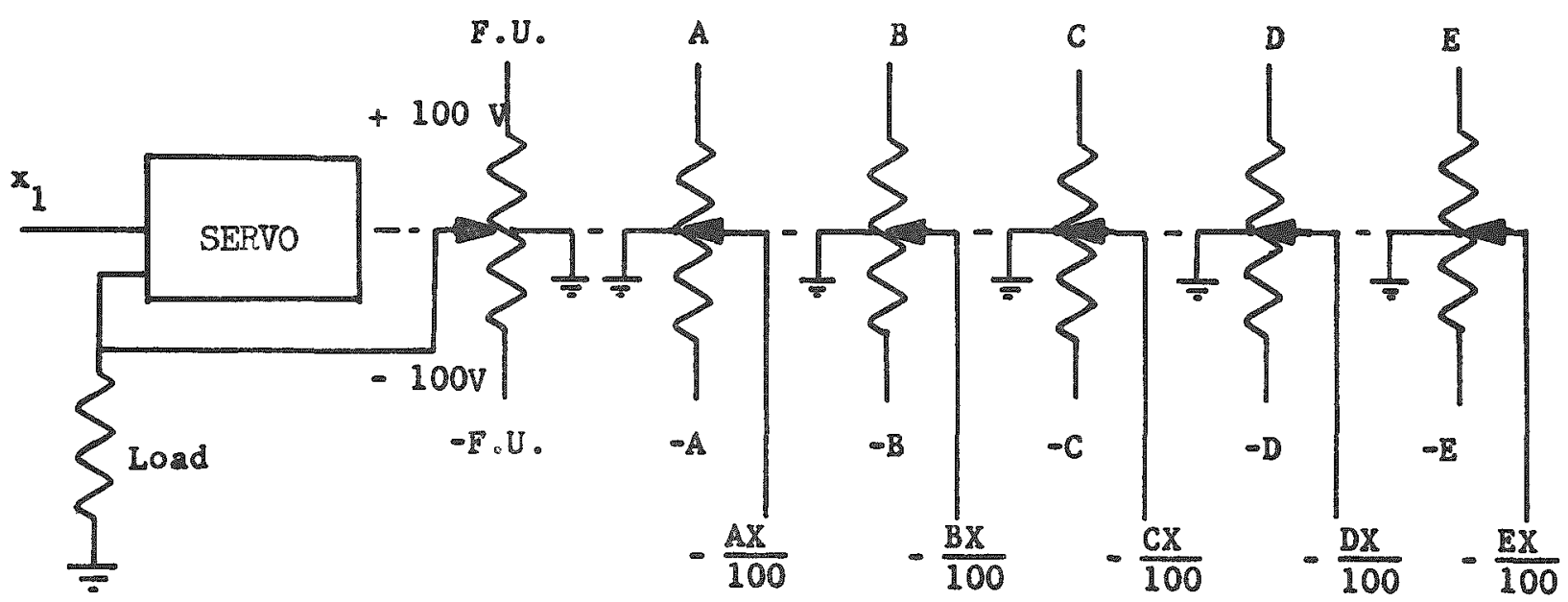

Figure $2 \pm$

SERVO MULTIPLIER POTS

The terminations of the multiplier pots and servo inputs on the prepatch panel are shown in Figure 25 .

The accuracy of the multiplier is determined by electrical errors, such as the gain setting, weak components, or components changing values, and mechanical errors, such as back-lash, friction, and motor torque.

When the servo is being used as a multiplier with " $\mathrm{X}$ " being applied to the input and the follow-up cup connected to +100 -volts and -100 -volts, the A cup connected with $a$ + voltage and a - voltage connected to $-A$, then 


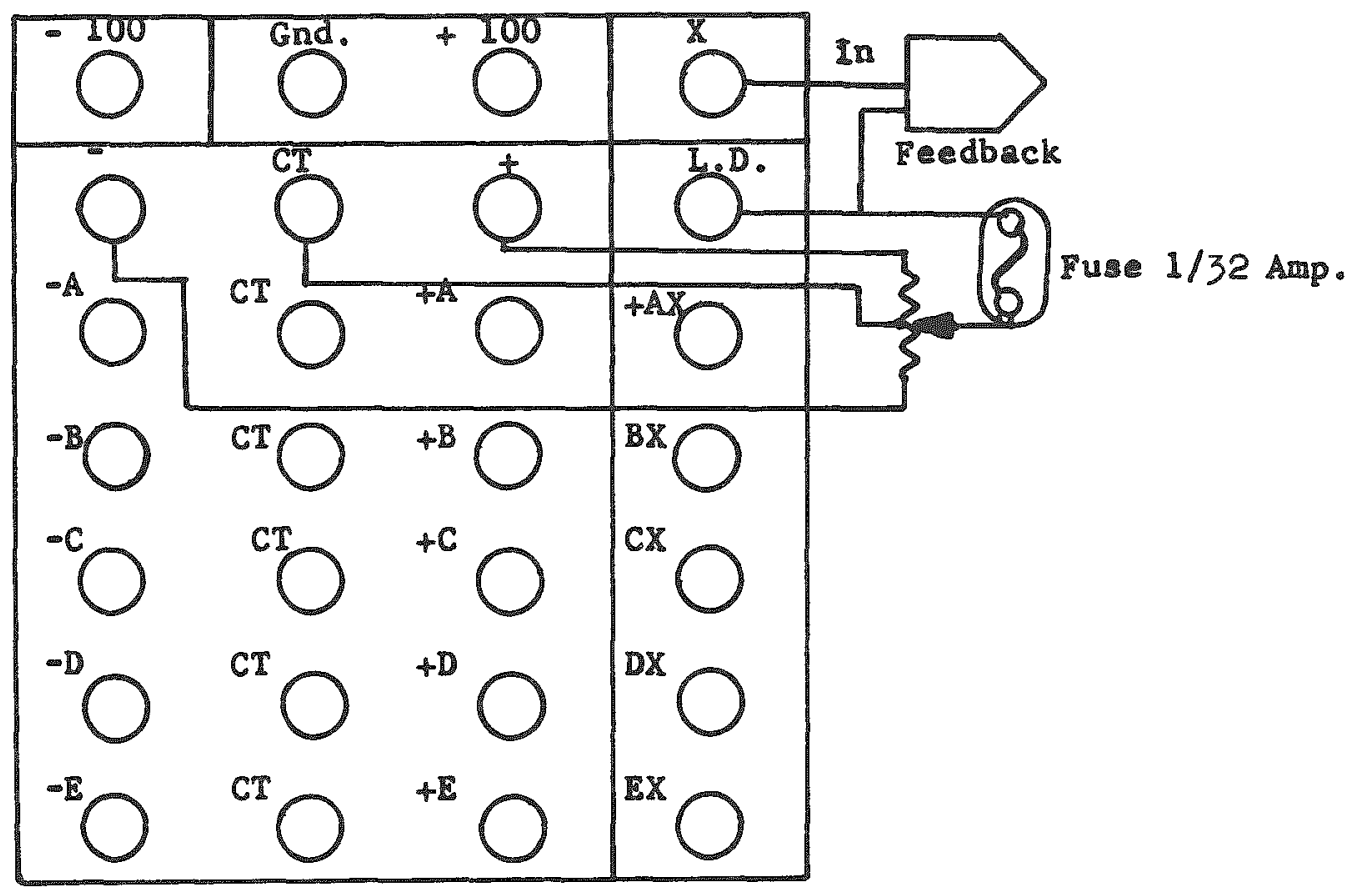

Figure 25

SERVO POT TERMINALS

the output of the $A$ cup will be $A X / 100$. If it is desired to obtain $+A X / 100$, then it is only necessary to reverse the inputs to the A cup. When the cups are connected for normal operation there is a sign inversion in the servo amplifier.

The servo's can also be used for division. The most common circuit used is shown in Figure 26. The only auxiliary equipment used is a highgain amplifier.

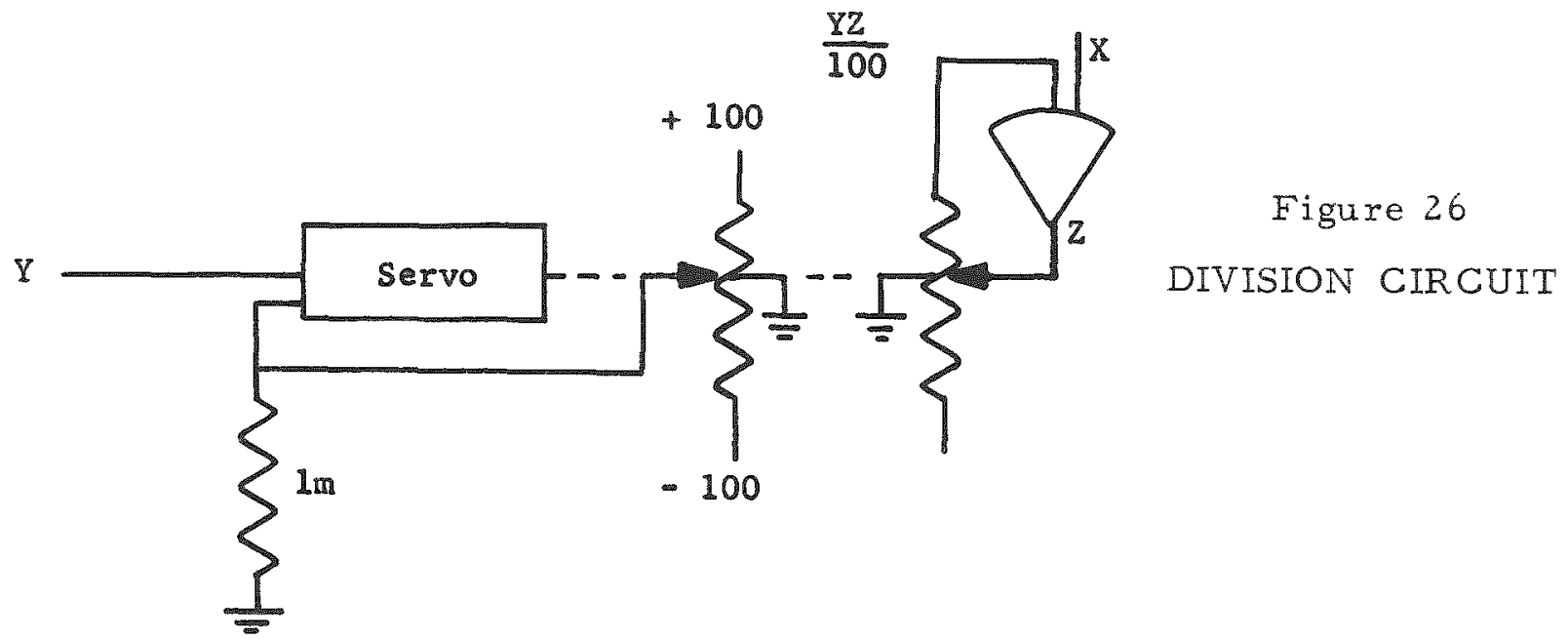


The multipliers are also used for obtaining square roots and for the computation of nonlinear functions. The computation of the nonlinear functions is accomplished by using the tapped pot (17 taps) provided in some of the multipliers in conjunction with the "Pot Padding" units. The tapped pot permits the variation of the voltage gradients necessary to produce the required function.

In operation of the servos there is some loading on all of the pots except the follow-up pot. This loading is caused by connecting the arms of the pots to the amplifier input resistors. Due to this loading effect it is necessary to externally patch a load resistor to the arm of the follow-up pot. The load resistor is connected between the terminal marked "LD" and ground. The value of the load resistor that is used is determined by the input resistor of the amplifier to which that arm is connected. If the arm of the pot is connected to the amplifier for a gain of 5 , then the load resistor for the follow-up pot is 0.2 megohm.

The outputs of all the cups of any multiplier being used can be individually selected by the signal selector pushbuttons and the output voltage displayed on the digital voltmeter.

NOTE: The outputs of all the pots from any one servo should be connected to the same value of load (i.e., gain of 5 ). 


\section{CLARY PRINTER}

The Clary Printer records the outputs of the Digital Voltmeter when it is monitoring the outputs of the components. The printer is a seven-column printer, with the three left-hand columns being used for the identifying codes and the four right-hand columns for recording the signal voltages. The printer will print manually one print at a time by depressing the print button on the control panel. It prints the output of the component that is selected by the signal selector pushbuttons. It will print, automatically, all of the outputs of the components. This is done by depressing the "AUTO" pushbutton in the left column of the signal selector switches and depressing 0,0 , in the next two columns. In the automatic mode the printer prints the out puts of trunks 1 thru 20, the arms of potentiometers $1-20$, summing points of integrators 1-28, outputs of amplifiers $1-56$, outputs of all servo cups, outputs of all electronic multipliers, outputs of D.F.G. $1-6$ and the signal on the cathode of diodes $1-4$.

The printer receives its signal from the Digital Voltmeter; therefore the Digital Voltmeter must be operating in order to use the Clary Printer. 


\section{DIGITAL VOLTMETER}

The digital voltmeter is designed for measuring and displaying the computer output voltages. The unit is a self-balancing digital potentiometer of the stepping switch type. It has a voltage range from zero to 99.99 volts DC with an accuracy of 0.01 volt The voltmeter consists of three sections: the power supply, the amplifier and the readout section. The power supply and the amplifier are mounted just above the temperature-controlled oven in the rear of the console, and the readout unit is located above the control panel of the console.

The digital voltmeter is an automatic potentiometer which produces a feedback voltage proportional to the unknown voltage input. The difference between the unknown input voltage and the feedback voltage causes a digital positioning system to adjust a voltage divider to reduce the difference or error voltage to zero. When this condition is achieved, the voltmeter is said to be balanced and the unknown voltage will be automatically displayed on the visual readout unit

The digital voltmeter will accurately follow an increase in the input signal, if the rate of change of the input signal does not exceed 10 millivolts in $\frac{1}{60}$ th of a second. Since the stepping swatches are undirectional, they will follow a decreasing input if the rate of change does not exceed 10 millivolts in $\frac{9}{60}$ th of a second The readings are displayed on one-inch luminous numerals.

The block diagram of the digital voltmeter is shown in Figure 27.

The polarities of the voltages that are being displayed on the digital voltmeter are fixed automatically and are shown in the left column in the readout unit.

There are only two controls for the voltmeter They are the gain control and the hum control: both are located on the amplifier chassis.

All of the calculated attenuator settings, initial condition voltages and the input voltages are set by use of the voltmeter; therefore it must be operating properly in order to obtain accurate results on the computer.

When first switching the computer on, it is necessary to wait 30 seconds before using the voltmeter, due to the time delay in the amplifier. This is for protection of the thyratron tubes 


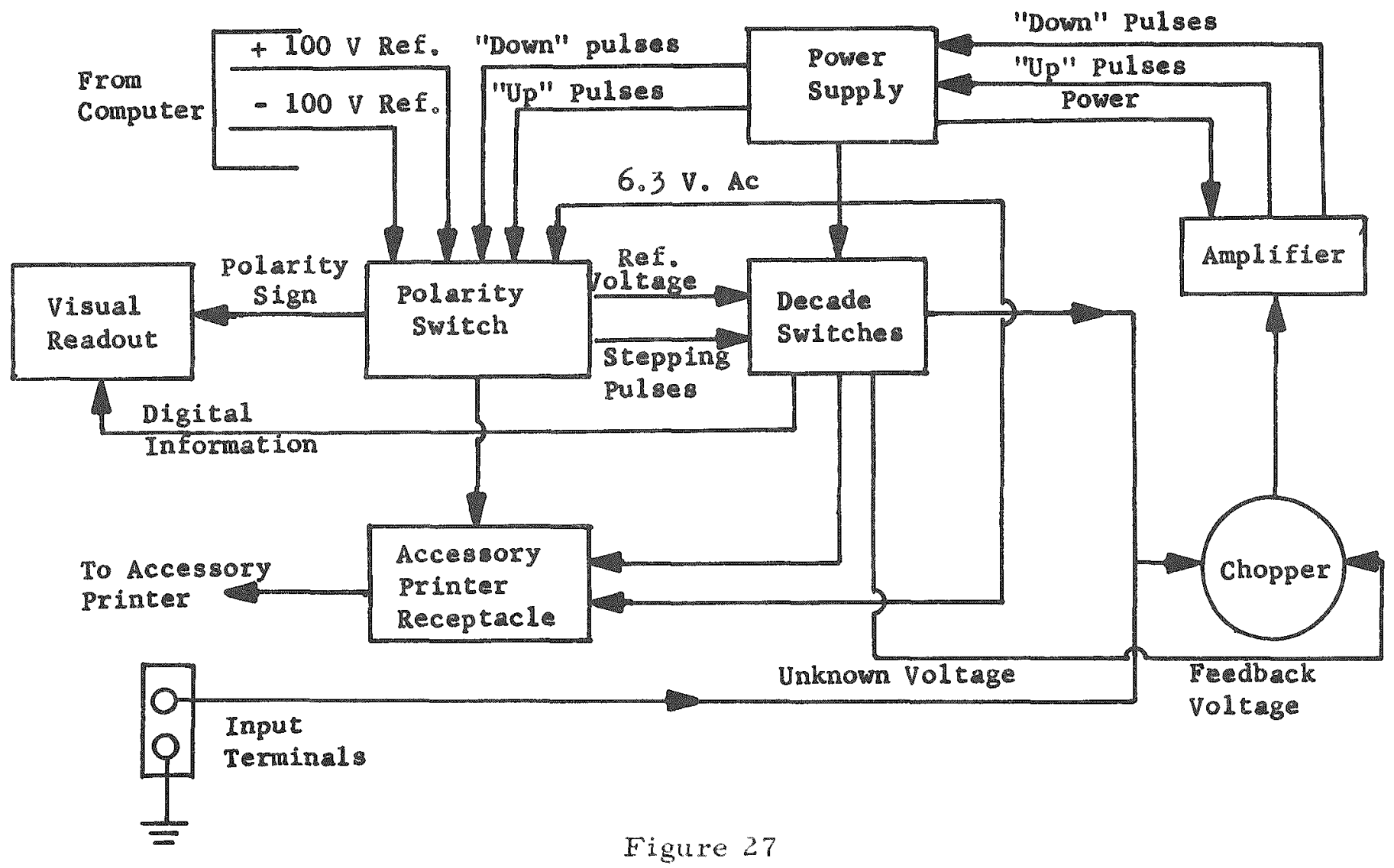

BLOCK DIAGRAM OF DIGITAL VOLTMETER 


\section{OSCILLOSCOPE}

The laxge-screen oscilloscope is a precision device that is used for the visual representation of low-frequency waveforms and data. Over the frequency range from DC to $20 \mathrm{kc}$ on the vertical axis and DC to $10 \mathrm{kc}$ on the horizontal axis, the linearity is within $1 \%$ and the measuring accuracy is $\pm 2 \%$ of the absolute full-scale value. The unit has a time base for driven and recurrent sweep operation that provides sweep from 10 microseconds per inch to 0.1 second per inch.

The input to the scope is brought out on the lower section of the pre-patch panel and the panel located below the scope's control panel.

On the left side of the scope control panel are the inputs to the vertical amplifier. The vertical amplifier controls, the sensitivity and the multiplier, and the DC balance control are located next to the vertical amplifier input binding posts. The DC balance control provides a means for balancing the deflection amplifier so that no spot shift results from rotation of the multiplier control. The horizontal axis controls that perform the same functions, only for the horizontal amplifier, are located on the right side of the scope control panel. The controls for the sweep circuits include the milliseconds-per-inch control that provides sweep ranges of $10,1,0.1$ and 0.01 milliseconds perinch. The off position of this control shorts the sweep capacitor so that the sweep for the scope can be driven from an external source. The multiplier is the vernier control. The sweep magnifier makes available a 2 to 1 increase in sweep speed by doubling the amplitude of the sweep waveform that is applied to the input of the horizontal amplifier. The sync selector switch permits the selection of internal, external or line sync of plus or minus polarity. The sync amplitude controls the operation of an amplitude comparator to determine the point on the positive or negative ex cursion of the signal which triggers off the sweep.

The sawtooth circuit provides a 7.5-volt peak-to-peak sawtooth out put at an internal impedance of approximately $70 \mathrm{k}$.

The Driven Recurrent switch permits a choice of driven sweep or nonsynchronizable free-running sweep. 


\section{SANBORN RECORDER}

The Sanborn recorder is a direct-writing instrument that uses the D'Arsonval galvanometers to drive a writing arm which wipes a hot-wire ribbon stylus across the paper while the paper is moving across a knife edge writing platen. The recorder has four separate channels, making it possible to record four different variables simultaneously. The recordings are permanent, instantaneous and in rectangular coordinates.

The inputs to the recorder are brought out on the pre-patch panel just below the row of attenuator terminals and approximately in the center of the board. The cables that connect the recorder to the computer are connected to the plugs in the floor and into either the front or the rear of the recorder amplifiers.

The green Sanborn permapaper that is used is four channels wide and each channel is five centimeters wide. The paper-drive motor will drive the paper at one of nine different speeds from 0.25 millimeter per second to 100 millimetersper second. The motor "off - on" $\mathrm{switch}$ and the clutch for the drive mechanism are located on the left of the control panel. The paper scale on the side of the control panel indicates the number of feet left in the roll of paper.

The controls on the front panel of the amplifiers include an attenuator control, which is a twelve-position control that inserts fixed attenuator factors into the input circuit. The calibration of this control is in volts per centimeter from 0.1 to 100 . In the calibrate position of this control the stylus should be adjusted for two centimeters of deflection by the gain control. The other position of the attenuator is the "OFF" position, which disconnects the inputs to the amplifier. The positioning control sets the baseline position of the stylus. The gain control sets the gain of the channel so that the sensitivity values stated on the attenuator are accurate. The stylus temperature control determines the amount of heat in the galvanometer for the optimum trace density. The balance control is a screwdriver adjustment that compensates for the residual unbalance of the amplifier. The balance of the amplifier is accomplished by turning the attenuator control to the "OFF" position and then, while rotating the gain control, adjusting the balance control until there is no movement of the stylus.

The fifth stylus of the recorder, located on the right side of channel

number four, is for the timer and marker signals. The timer signals are a series of pulses at one-second intervals that continue for as long as the timer circuit is closed. The marker signal is a signal pulse and marks only as long as the spring-loaded switch is held in the marker position. 


\section{VARIPLOTTERS}

The solution of the problems that are solved on the computer is plotted on rectangulax coordinate graph paper by means of a "Variplotter." The variplotter is an $X-Y$ plotter that can be used to plot two variables simultaneously or to plot one variable against time. There are two types of plotting tables used in this installation. The model $1100 \mathrm{D}$ is a portable table with one arm and a pen and a plotting surface of 10 by 15 inches. The model $205 \mathrm{~N}$ is an upright plotter with a plotting area of 30 by 30 inches. The $205 \mathrm{~N}$ plotter has two sets of pens, making it possible to record two independent sets of inputs of $X$ and $Y$ simultaneously, both using the full plotting surface. This model employs an automatic pen interchange circuit so that the pens will interchange functions whenever they intersect. The graph paper is held to the vinyl plotting surface by means of a vacuum.

The inputs to the plotting tables are brought out on the pre-patch panel and from the pre-patch panel they are wired to the connectors on the floor: from the floor they are connected by means of a cable to the data connector plug on the plotting table that is being used. The inputs to the model $205 \mathrm{~N}$ are plugged into the data panel in the lower section of the cabinet. The plugs on the floor connectors are marked to correspond to the number on the prespatch panel.

The input voltages to the variplotters are applied to the servo am plifiers The servo amplifier compares the brush voltage with the signal input voltage and supplies the motor with a voltage of such a phase that the resultant rotation of the servo motor will position the brush to keep the difference between the signal and brush voltage at a minimum. There are separate servo amplifiers for the pen circuit and the arm circuit The controls of the servo amplifier are the gain control that sets the bias on the second stage of amplification in the servo amplifier, and the damping control which determines the amount of feedback that is being applied to the chopper to be compared with the input voltage.

The scale-factor control varies the magnitude of the DC voltage that is supplied to the feedback potentiometer. This control provides a sensitivity from 0.1 volt per inch to 20 volts per inch in steps of $0.1,0.2$, $0.5,1.0,2.0,5.0,100$, and 20.0 volts per inch. There is a scale-factor control for the pen and a control for the arm. The parallax controls perm mit the positioning of the arm or pen anywhere on the paper. This control varies the level of the voltage applied across the feedback potentiometer, but not the magnitude.

The Stand-By Operate switch completes the circuit for the inputs to be applied to the servo amplifiers. In the Stand.By position the arm is po= sitioned on the right side of the table and the pen is positioned in the center of the plotting table. The Pen Up-Down completes the circuit so that a volt age is applied to a solenoid which raises the pen. 
The ink used for plotting is either the black India ink or the green Esterline ink. If it is desirable to make several copies of the plotted graph, it is recommended that the black India ink be used, since it will reproduce much more clearly. 


\section{EUNCTION GENERATOR KIT}

The function generator kit is used in conjunction with the model $1100 D$ variplotter for the generation of a signal proportional to the amplitude of a curve that is a continuous function of $X$.

The function generator kit consists of a curve follower head or "prck-up" head for tracing the curve and an oscillator that supplies the pick-up head with a reference voltage and also supplies the curve with an excitation voltage.

In setting up the function generator kit, the curve that is to be generatedistraced on the graph paper with a very fine conducting wire (No. 40). After placing the curve on the variplotter, the left end of the curve is connected to the oscillator by coax cable and the other end of the curve is grounded. The pick-up head is placed in the inkwell position on the pen assembly. The output of the function generator, the ground connections, and the \pm 100 -volt references are connected to the compute $r$ by the prempatch panel. The gain and the damping of the servo amplifier will have to be readjusted due to a difference in mass between the curve pick-up head and the pen inkwell assembly.

The oscillator supplies an excitation voltage to the curve, causing a field to appear about the conductor. The field on one side of the conductor is 180 degrees out of phase with the field on the opposite side of the conductor. When the pick-up head is centered on the conductor, the voltages induced in the coil will cancel and no current will flow in the external load. When the head is deflected to either side of the conductor, the field on that side induces a voltage into the coils this voltage is rectified by one of the crystals.

The rectified current of the crystals flows through the external load resistor of the head and appears at the input of the servo amplifier as an error voltage. The polarity of this error voltage is dependent upon the direction that the head was deflected from the conducting wire. The error voltage is then fed to the servo mechanism which positions the pickup head in such a manner as to reduce the amplitude of the error and to keep the head centered on the conducting wire.

The controls of the function generator kit include the bias control that sets the reference voltage to the required level The bias control is adjusted for maximum sensitivity with minimum offset of the head. The frequency control determines the operating frequency of the oscillator, which should be $470 \mathrm{kc}$. This control is a coil and is adjusted for maxim mum sensitivity of the curve pick-up head. 